\title{
Ambienti digitali per lo sviluppo delle competenze trasversali nella didattica universitaria*
}

\section{Concetta La Rocca - Massimo Margottini Rosa Capobianco}

Università degli Studi "Roma Tre», Dipartimento di Scienze dell'Educazione

doi: 10.7358/ecps-2014-010-laro

concetta.larocca@uniroma3.it massimo.margottini@uniroma3.it

rosa.capobianco@uniroma3.it

\section{DIGITAL ENVIRONMENTS FOR THE DEVELOPMENT OF TRANSVERSAL COMPETENCES IN UNIVERSITY EDUCATION}

\section{Abstract}

International studies show that university teaching is making increasing use of digital environments to integrate traditional teaching. In this paper we consider the contribution that can be made by online environments to develop students' positive and proactive attitudes with respect to the academic curriculum in order to reduce student drop-out, encourage greater regularity in their academic career, develop transversal competences and promote student guidance. In particular, we have observed the online activities in academic year 2013/2014 carried on by students of General Didactics, a degree course in the Department of Educational Sciences of "Roma Tre» University. These activities include the realization of two online practical classes: completion of the QSA (Questionnaire for Learning Strategies); participation in a cooperative online learning exercise. In both experiences we analyzed the procedures and outcomes. We observed that, through the QSA, students reflected on their learning strategies and on the importance of planning their university commitments: a positive factor is the high percentage of students who took the exam successfully in the first session (approximately 68\%). In addition, the questionnaire data clearly show that

* Questo articolo è il risultato del lavoro congiunto dei tre autori. In particolare sono da attribuire a C. La Rocca e M. Margottini i parr. 1 e 5; a M. Margottini i parr. 2 e 3; a R. Capobianco il par. 3.2; a C. La Rocca il par. 4. 
students found that group work helps to develop cognitive and meta-cognitive competences as well as relationship competences. In particular, we found that in the General Didactics examination the students who had experienced cooperative learning obtained higher grades, on average, than those who did not, and that the former group had less "sigma" compared to the latter.

Keywords: Cooperative learning, Freshman guidance, Online environments, QSA (Questionnaire for Learning Strategies), Transversal competences.

\section{INTRODUZIONE E PRESENTAZIONE DEL PROGETTO}

L'esperienza didattica che si presenta in questo lavoro intende rispondere alle sollecitazioni espresse dalle direttive europee che di fatto invitano i sistemi di istruzione ad intervenire in maniera operativa per sviluppare competenze generali e trasversali, al fine di formare giovani adulti che possano affrontare il mondo del lavoro con una maggiore competenza e adattabilità professionale. Naturalmente questa prospettiva riguarda in generale le scuole di ogni ordine e grado; il presente studio pilota affronta il tema in ambito universitario, e riporta in particolare gli esiti dell'intervento formativo avvenuto nell'a.a. 2013/14 a cui hanno partecipato gli studenti dell' 'Insegnamento di Didattica Generale», attivo presso il Corso di Laurea in Scienze dell'Educazione nel Dipartimento di Scienze della Formazione dell’Università «Roma Tre».

L'idea generale, che ha determinato l'esigenza di dare avvio a questa iniziativa, può essere espressa nei seguenti punti:

a. L'adozione di strategie didattiche integrate che sviluppino negli studenti competenze generali ed autoregolative possono favorire una disposizione positiva e propositiva verso il percorso di studi per completare la preparazione accademica, così da arginare l'abbandono, favorire l'orientamento in itinere e facilitare l'ingresso e la permanenza nel mondo del lavoro.

b. Le tecnologie di rete possono essere considerate un supporto elettivo agli scopi appena descritti, poiché consentono di costruire e mantenere nel tempo attività e contatti che la didattica tradizionale in presenza spesso non può garantire. Si tratta di un uso delle tecnologie che assume una "curvatura» funzionale al progetto didattico. Ed in tal senso l'ambiente online, che si basa su una piattaforma e-learning Moodle 2.x, è opportunamente "costruito" attraverso l'integrazione di altri strumenti che di volta in volta possono risultare funzionali alla realizzazione delle attività didattiche. Ad esempio per la somministrazione del QSA e relative elabo- 
razioni si è fatto uso della piattaforma LimeSurvey che meglio si presta alla gestione di questionari complessi e relative elaborazioni.

Nello specifico le linee di sviluppo generali sopra descritte sono state articolate nelle seguenti ipotesi che hanno determinato l'intervento didattico di cui si dà conto in questo studio:

1. La compilazione online del QSA consente agli studenti di riflettere efficacemente sul proprio modo di affrontare lo studio dal punto di vista organizzativo, cognitivo, emozionale e motivazionale per regolare di conseguenza il proprio comportamento nell'apprendimento.

2. La partecipazione ad attività di cooperative learning online favorisce lo sviluppo di abilità relazionali e meta-cognitive che possono incidere positivamente sull'esito della carriera accademica.

Come si è anticipato, il contesto in cui viene svolta la ricerca empirica è l' «Insegnamento di Didattica Generale» che è stato ritenuto adeguato allo scopo per i seguenti motivi:

- La struttura in modalità blended: il corso da 9 CFU (Crediti Formativi Universitari) è strutturato in un corso base da 6 CFU che prevede lo svolgimento di lezioni in aula e attività laboratoriali per 3 CFU da svolgersi online, sulla piattaforma e-learning di Dipartimento all'indirizzo http:// formonline.uniroma3.it/.

- La coerenza del tema dell'indagine con i contenuti disciplinari trattati nel corso.

- Il fatto che il corso di Didattica Generale è rivolto a studenti del primo anno che, nella loro posizione di matricole, mostrano spesso un certo disorientamento nella comprensione delle dinamiche accademiche, disagio accresciuto dal grande numero di studenti frequentanti (oltre 300).

- La tipologia degli studenti che presentano spesso una preparazione inadeguata ad affrontare il percorso di studi, circa il $20 \%$ non ha superato il test di accesso e risulta pertanto iscritto con Obblighi Formativi Aggiuntivi da evadere nel primo anno di corso. Si consideri inoltre che la percentuale di studenti che abbandonano gli studi nel primo anno o risultano in ritardo nella carriera è piuttosto alta.

- La constatazione, derivata anche da esperienze precedenti, che le attività online, di compilazione del QSA e di cooperative learning consentono agli studenti non frequentanti l'opportunità di conoscere i colleghi e di organizzare con loro gruppi di lavoro attraverso le interazioni previste nei forum.

Per una migliore comprensione della struttura dell'indagine effettuata, sembra opportuno dare conto di alcuni elementi:

- La compilazione del QSA è obbligatoria per tutti gli studenti che seguono il corso di Didattica Generale, pertanto la prima parte (par. 3) del lavoro di ricerca riguarda l'intera popolazione di studenti. Hanno compilato il QSA 327 studenti. 
- La partecipazione alle attività collaborative è facoltativa (par. 4); per gli studenti che scelgono di svolgere i lavori di gruppo è obbligatoria la compilazione del questionario (barometro) che rileva le opinioni degli studenti sull'esperienza. A posteriori si è però constatato che i questionari somministrati in itinere sono risultati 63 e 67 quelli in uscita, a fronte di un numero complessivo di 78 studenti che hanno partecipato alle attività di gruppo. Ciò è accaduto perché gli studenti si sono aggiunti progressivamente alle attività in piattaforma, probabilmente attratti da una esperienza che inizialmente non era sembrata loro interessante. Poiché i questionari sono anonimi non è stato possibile pareggiare i numeri in ingresso e in uscita.

- Entro la prima sessione d'esame hanno sostenuto l'esame 228 studenti iscritti nell'a.a. 2013/14; tra questi risultano 78 studenti iscritti nell'a.a. 2013/14 che hanno partecipato alle attività di gruppo.

La descrizione dello svolgimento delle specifiche attività intraprese per la somministrazione del QSA e per l'organizzazione delle attività di cooperative learning, e i relativi esiti, costituiscono l'argomento svolto rispettivamente nei parr. 3 e 4 . Pur nella condivisione degli scopi formativi, degli obiettivi generali e della strategia didattica complessiva, si è scelto di presentare separatamente le due esperienze per mantenere inalterato l'impianto metodologico che ne ha caratterizzato la sistematicità.

\section{LA DIDATTICA UNIVERSITARIA PER PROMUOVERE COMPETENZE}

La tendenza dei sistemi d'istruzione e formazione a focalizzare l'attenzione sugli esiti formativi in termini di conoscenze e competenze acquisite è andata sempre più consolidandosi nel corso degli ultimi decenni. Tendenza che s'inquadra nel cosiddetto Processo di Bologna, attraverso il quale l'Europa si è posta l'obiettivo di armonizzare i titoli di livello terziario degli stati membri, ponendo in rilievo la centralità del soggetto in apprendimento con una particolare attenzione sui risultati, ed in particolare sul profilo di competenze acquisite in uscita dai percorsi di studio e sulle modalità didattiche e disciplinari di raggiungimento dei risultati stessi (Serbati \& Zaggia, 2012).

La strategia europea di focalizzazione sulle competenze che gli studenti devono possedere in uscita dai sistemi formativi vede ulteriori momenti di specificazione e qualificazione con l'adozione del Framework for the Qualification of the European Higher Education Area (EHEA, 2005) ed in particolare con la definizione dei cosiddetti descrittori di Dublino, che vogliono declinare i risultati di apprendimento attraverso cinque descrittori di carattere generale e posti in ordine tassonomico: conoscenza e capacità di comprensione, cono- 
scenza e capacità di comprensione applicate, autonomia di giudizio, abilità comunicative e capacità di apprendimento.

Parallelamente in una prospettiva lifelong learning, con risoluzione del 2008, la Commissione Europea approva lo European Qualification Framework for Lifelong Learning (EQF) che ancor più, in una prospettiva di educazione permanente, rimarca ulteriormente il rilievo di un processo di apprendimento per competenze.

A dare ulteriore importanza a questa prospettiva svolge un ruolo determinante il rapporto con il mondo del lavoro che lamenta sul versante della domanda una scarsa rispondenza tra le qualifiche conseguite all'interno del sistema formativo e gli skill ${ }^{1}$ richiesti dal mercato del lavoro. Con il termine mismatch s'indica, appunto, il mancato allineamento fra la domanda di competenze espressa dalle imprese e l'offerta delle stesse da parte dei lavoratori. Le ragioni del disallineamento possono essere ricondotte ad una molteplicità di fattori: dalle asimmetrie informative, alla rigidità dell'offerta, all'internazionalizzazione, alle rapide evoluzioni tecnologiche e il fenomeno è oggi acuito dal livello di turbolenza e dinamismo del tessuto economico-industriale internazionale. «L'aumentata dinamicità dell'ambiente richiede alle imprese una nuova, e per certi versi originale, attenzione alla definizione di percorsi di crescita cumulativa del proprio stock di capitale umano. L'obiettivo è rappresentato dall'esigenza di un continuo aggiustamento fra competenze richieste, in costante evoluzione, e competenze possedute dai dipendenti, anch'esse mutevoli nel tempo» (Cainarca \& Sgobbi, 2001).

In primo luogo, anche a seguito dell'enorme varietà degli skill normalmente esercitati in qualsiasi attività professionale, si pone il problema di selezionare una gamma contenuta di abilità e competenze capaci di cogliere eventuali mismatch sottolineando anche che spesso le imprese concentrano il proprio interesse verso gli skill di tipo soft, quali comunicazione interpersonale, attitudine verso il lavoro, capacità di lavorare in gruppo, in misura almeno pari rispetto agli skill di natura cognitiva.

Con l'obiettivo di sviluppare esperienze e strumenti che consentano di mettere a sistema i quadri espressi dal Processo di Bologna e dallo European Qualification Framework, la Commissione Europea ha promosso il progetto Tuning che ha posto al centro delle proprie azioni la promozione di un modello competence-based learning con una declinazione delle competenze in uscita in specifiche e generali. E sebbene sottolinei il rilievo delle conoscenze e competenze strettamente specifiche e disciplinari per ciascun corso di lau-

1 Esistono in letteratura molteplici definizioni alternative al concetto di skill, tra le molte una sintetica e al tempo stesso abbastanza generale considera gli skill come "capacità di svolgere i compiti e i doveri di un lavoro in modo competente» (Elias \& McKnight, 2001).

ECPS Journal - 10/2014

http://www.ledonline.it/ECPS-Journal/ 
rea universitario, evidenzia con maggiore enfasi il fatto che dovrebbe essere dedicato maggior tempo e attenzione allo sviluppo di competenze generali e trasferibili, sottolineando come quest'ultima componente sia sempre più rilevante per preparare gli studenti a realizzare il proprio futuro nella società in termini di occupabilità e cittadinanza, si tratta quindi di competenze di natura trasversale. Distingue pertanto tre tipi di competenze generali o, se si vuole, trasversali: le competenze strumentali intese come capacità cognitive, metodologiche, capacità tecnologiche e competenze linguistiche; le competenze interpersonali come abilità individuali e sociali per le interazioni sociali e la cooperazione; le competenze sistemiche come capacità di integrazione in sistemi complessi di comprensione, sensibilità e conoscenza, previa acquisizione delle competenze strumentali e interpersonali necessarie.

In ambito nazionale con la sperimentazione TECO (Test sulle competenze effettive di carattere generalista dei laureandi italiani, 2012/13), condotta dall'ANVUR (Agenzia Nazionale del Sistema Universitario e delle Ricerca) si avvia un processo che mira a conoscere la qualità degli apprendimenti realizzati durante il percorso di formazione in termini di competenze generaliste possedute dagli studenti al momento del rilascio del titolo universitario, anche nella prospettiva di auto-valutazione, valutazione e accreditamento dei corsi di studio e delle sedi universitarie (AVA - Auto-valutazione, Valutazione periodica, Accreditamento). Con il test TECO si valutano quindi competenze trasversali che «sono quelle in grado di garantire flessibilità e adattamento degli individui ai cambiamenti professionali e personali che intervengono durante le fasi del ciclo vitale. Oltretutto queste competenze non sono monitorate né valutate dagli atenei proprio perché non sono oggetto di uno specifico insegnamento ma fanno parte di quel bagaglio intangibile che ogni docente dovrebbe trasmettere attraverso l'insegnamento della propria materia" (Rapporto TECO-ANVUR, 2014).

Gli esiti del test evidenziano un sostanziale allineamento dei risultati dei nostri laureandi a quello di altri paesi ed in particolare di un analogo campione di laureandi di college americani, i punteggi risultano sostanzialmente allineati, tuttavia si evidenzia una significativa differenza. Una superiore efficacia e tecnica di scrittura, maggiore capacità di argomentazione e lettura critica dei laureandi italiani ma inferiore qualità nel ragionamento scientifico-quantitativo.

Nel confronto interno emergono invece due ordini di differenze: da un lato tra gruppi di lauree scientifiche e gruppi di lauree umanistiche, dall'altro tra atenei del Centro-Nord (esclusi quelli dell'area romana) e atenei del SudIsole. Il punteggio medio di studenti con identiche caratteristiche osservate è infatti più alto per i gruppi di lauree scientifiche (in particolare per il gruppo Matematica, Fisica e Statistica) e per gli Atenei del Centro-Nord, e più basso per i gruppi di lauree umanistiche (in particolare per il gruppo Beni culturali e il gruppo Formazione) e per gli atenei del Sud-Isole. Dai dati del Rappor- 
to TECO emergono, quindi, significative differenze non solo territoriali ma anche d'indirizzo di studio che finiscono poi per connotare sul mercato del lavoro le cosiddette «lauree deboli» ossia quelle meno richieste.

\section{IL QSA PER L'AUTO-VALUTAZIONE DELLE COMPETENZE STRATEGICHE}

In un'ottica di promozione di competenze strategiche per dirigere se stessi nello studio e nel lavoro (Pellerey, 2006), suscita un notevole interesse la possibilità di attivare, mediante appositi strumenti, un processo di riflessione e di autovalutazione dei livelli di competenza raggiunti nella dimensione autoregolativa dell'azione e dell'apprendimento. In primo luogo, perché questo rappresenta uno step indispensabile nella prospettiva dell'avvio di percorsi autoformativi volti allo sviluppo delle competenze di auto-direzione. Secondariamente, perchè la presa di coscienza da parte del singolo, riguardo alle risorse ed ai livelli di padronanza raggiunti, costituisce una condizione imprescindibile per l'attivazione dell'energia motivazionale che guida tutti i processi di apprendimento.

Si può affermare, inoltre, che la competenza autoregolativa assuma un carattere autogenerativo: non solo rende possibile lo sviluppo di nuove conoscenze e abilità ma anche l'accrescimento progressivo della capacità di governare in modo sempre più sofisticato i propri processi di apprendimento (Pellerey, 2006). Studi relativamente recenti (Zimmerman, 2000, 2001 e 2002) hanno dimostrato che i soggetti più autoregolati hanno livelli elevati del senso di auto-efficacia, quindi, un elevato grado di fiducia riguardo alla loro capacità di raggiungere gli obiettivi prestabiliti; in secondo luogo, si caratterizzano per l'intenzione di conseguire un reale apprendimento personale in termini di competenze sviluppate (non in vista di ricompense estrinseche); ed hanno un interesse intrinseco per i contenuti o le attività oggetto dell'apprendimento.

Le problematiche connesse alla valutazione delle competenze strategiche di tipo autoregolativo, sono state oggetto di studio da parte di vari autori e rappresentano un ambito di indagine su cui molto si è discusso e si continua a dibattere. Innanzitutto, come sostiene Pellerey (Bay, Grzadziel, \& Pellerey, 2010), per ottenere un giudizio valutativo sulle competenze che sia adeguatamente valido e verosimile, è necessario fare riferimento alla metodologia della «triangolazione». Essa si fonda sul ricorso ad una molteplicità di approcci valutativi: l'osservazione della prestazione nel momento della messa in atto da parte del soggetto, l'auto-valutazione, l'analisi finale della qualità della performance e dei risultati conseguiti. È necessario, inoltre, ricordare che i questionari di auto-valutazione si rifanno alla dimensione soggettiva 
della competenza che si lega alle particolari modalità mediante le quali ogni individuo fa sue ed organizza conoscenze ed abilità, alle sfumature affettivomotivazionali e di significato sulla base delle quali queste vengono integrate tra loro, all'auto-percezione dei propri livelli di padronanza.

Anche le indagini PISA (2000-2009), promosse dall'OCSE, hanno sottolineato la necessità di valutazione di competenze di natura trasversale, definite «cross-curricolari». Accanto alle capacità di comprensione della lettura di testi scritti e di quelle matematiche e scientifiche, si è ritenuto di sottoporre ad un'adeguata analisi anche le competenze cosiddette "cross-curricolari», trasversali alle singole discipline. Nello specifico, sono state oggetto di indagine le strategie cognitive (la reiterazione o memorizzazione, l'organizzazione e l'elaborazione) e meta-cognitive (i processi di monitoraggio e valutazione della propria attività durante lo studio), le abilità connesse allo studio (le abilità organizzative), le cognizioni riferite al sé (il concetto di sé, le autoattribuzioni), la motivazione e l'impegno nello studio.

Nel presente lavoro si fa riferimento, in particolare, alla utilizzazione di uno strumento autovalutativo elaborato e validato da Michele Pellerey e collaboratori: il Questionario sulle Strategie di Apprendimento (QSA) (Pellerey, 1996). Dalle precedenti ricerche, Pellerey individua un quadro di competenze strategiche che possono essere considerate alla base della capacità di autodirigersi, sia dal punto di vista «strategico» della auto-derminazione, sia da quello "tattico" della auto-regolazione. Il quadro può essere sintetizzato come segue.

Competenze strategiche messe in atto per capire e ricordare. Come noto, lo studio e l'apprendimento chiamano in causa azioni sequenziali tra le quali quella della comprensione e della memorizzazione. Ogni individuo adotta strategie personali per interiorizzare nuovi contenuti ed integrarli nella struttura di conoscenze ed esperienze consolidate. La modalità prevalente di elaborazione dell'informazione che il soggetto adotta in modo duraturo nel tempo ed estende a compiti diversi è stata definita stile cognitivo (Boscolo, 1981). La letteratura psicologica e pedagogica, ha evidenziato la presenza di stili differenti, alla luce dei quali ogni individuo si approccia alle situazioni di apprendimento, ad esempio Kolb (1985) distingue gli stili in accomodatore, divergente, assimilatore, convergente. Nell'ambito dell'azione d'insegnamento è opportuno tener conto di ciò, non solo per attivare approcci didattici differenziati in relazione alle particolarità degli studenti, ma anche per motivare gli stessi all'adozione flessibile delle strategie cognitive più adeguate in relazione alla specificità del compito e delle situazioni.

Competenze strategiche relative alla disponibilità a collaborare nel lavoro e nell'apprendimento. $\grave{E}$ ormai assodato che l'interazione sociale assume un ruolo centrale nell'apprendimento, anche nell'ottica dello sviluppo di capacità di lavoro in gruppo. Questa constatazione è alla base dei più moderni approcci 
di cooperative learning fondati su una visione socio-costruttivista. Vari autori nel corso del passato hanno sottolineato tale aspetto. Piaget (1985) evidenzia che il conflitto sociocognitivo derivante dallo scambio reciproco tra pari genera nel singolo nuove modalità interpretative della realtà che gli consentono di superare la fase del cosiddetto "egocentrismo cognitivo». Secondo Vygotskij (1987) in qualsiasi età, lo sviluppo cognitivo necessita dell'interazione sociale per esplicarsi pienamente. Sotto la guida di un adulto o grazie alla collaborazione tra pari, è possibile sviluppare un insieme più complesso di abilità rispetto a quello che si può ottenere mediante l'apprendimento individuale.

Competenze strategiche nel comunicare e nel relazionarsi con altri. In ogni contesto formativo o professionale la capacità di esprimere in modo chiaro e preciso i propri pensieri, i propri stati d'animo, le proprie conoscenze, costituisce una risorsa necessaria per la realizzazione personale del soggetto e per lo sviluppo di relazioni sociali fondate sulla trasparenza e l'accettazione reciproca. In ambito scolastico è possibile incrementare questo tipo di competenze mediante la promozione di attività che stimolino il soggetto a motivare e ad argomentare le proprie convinzioni.

Competenze strategiche nel dare senso e prospettiva alla propria esistenza umana e lavorativa. Esse attengono alla conoscenza di sé, dei propri interessi, delle proprie potenzialità e aspirazioni. In un percorso educativo, le finalità formative dovrebbero integrarsi strettamente alla promozione nel discente della capacità di riflettere su stesso, di attribuire significato alla propria esistenza e di prefigurarsi un percorso mentale che lo guidi nella realizzazione dei suoi obiettivi di vita.

Percezione soggettiva di competenza. Come noto, i giudizi di auto-efficacia riguardo alle proprie capacità scolastiche o professionali influiscono sull'impegno e le energie attivati, sugli obiettivi perseguiti, sulla perseveranza dello sforzo nel conseguimento di una meta. Essi sono determinanti per lo sviluppo progressivo delle competenze, poiché motivano l'individuo ad affrontare compiti sfidanti e stimolanti, che promuovono la crescita personale (Bandura, 2000).

Stile attributivo e competenze strategiche nel gestire le attribuzioni causali. Quest'ultime spesso non vengono espresse esplicitamente o percepite consapevolmente ma rimangono a livello tacito. Diviene, pertanto, significativa un'azione formativa volta a promuovere la consapevolezza del soggetto riguardo al ruolo della mediazione cognitiva nell'inferenza delle possibili cause degli eventi (Weiner, 1985). Sarebbe importante, ad esempio, che lo studente fosse consapevole del carattere mutevole dell'intelligenza in relazione all'apprendimento ed all'impegno nello studio.

Competenze strategiche nel gestire forme accentuate di ansietà. La legge di Yerkes e Dodson (1908) evidenzia chiaramente che l'ansietà mantenuta su livelli di attivazione ottimali (Eustress) conduce il soggetto al raggiungimento di prestazioni efficaci, garantendo un'adeguata reattività, attenzione e 
concentrazione. Come noto, d'altro canto, stati ansiosi particolarmente accentuati giocano un ruolo controproducente nei processi di apprendimento, inibendo le capacità cognitive. Nel corso del tempo, in ambito psicologico, sono state proposte diverse strategie utili a supportare il soggetto nella gestione efficace e produttiva della propria emotività.

Competenze strategiche nel gestire se stessi nel lavoro e nell'apprendimento: auto-regolazione e volizione. Come già affermato nelle pagine precedenti, l'attivazione di comportamenti organizzati nell'ottica del raggiungimento di un determinato fine chiama in causa tanto i processi motivazionali quanto quelli volitivi di controllo dell'azione. «I processi volitivi possono essere considerati come una parte del più ampio sistema dei meccanismi di auto-regolazione, e riguardano specificatamente la focalizzazione della propria attenzione ed impegno verso un obiettivo» (D’Alessio, Laghi, \& Pallini, 2006).

Competenze strategiche nell'affrontare situazioni sfidanti o pericolose e nel decidere: "coping». Le strategie di coping possono esser definite come «'insieme degli sforzi comportamentali e cognitivi, volti alla gestione di specifiche richieste esterne e/o interne, valutate come situazioni che mettono alla prova o che in ogni caso eccedono le risorse di una persona» (Lazarus \& Folkman, 1984). Esse sono centrate sulla dimensione cognitiva, nel momento in cui il soggetto tenta di attivare specifiche modalità di risoluzione dei problemi e su quella emotiva, quando si impegna nella gestione dei sentimenti negativi connessi alla situazione stressante.

La scuola secondaria superiore (che costituisce il primo vero snodo orizzontale dei percorsi d'istruzione nel sistema educativo italiano) rappresenta l'ambito privilegiato per la stesura da parte dello studente di un bilancio delle risorse possedute per affrontare efficacemente il percorso futuro.

Il QSA si propone come strumento di auto-valutazione, nella fase iniziale del percorso secondario, volto a favorire la riflessione e l'acquisizione di una maggiore consapevolezza sul livello di competenza raggiunto in alcuni degli aspetti necessari a procedere efficacemente nello studio e nel lavoro.

Sebbene pensato per essere impiegato prevalentemente nella scuola superiore il QSA è stato già applicato con successo anche in ambito universitario (Pellerey, 1996; Pellerey et al., 2013). Tuttavia, in questo contesto, è più evidente la necessità di coinvolgere lo studente in un percorso autoformativo volto alla presa di coscienza ed alla valorizzazione delle competenze sviluppate nell'ambito dell'auto-regolazione e dell'auto-determinazione (Pellerey et al., 2013). Rispetto a quest'ultima dimensione, assume un ruolo centrale la capacità di definire un progetto esistenziale, di studio, di lavoro e di attribuire significato alle proprie scelte e condotte. Il QSA costituisce uno strumento che ha ottenuto significativi riscontri nelle varie sperimentazioni, grazie alla semplicità di utilizzo, ai brevi tempi di somministrazione, alle ricadute sul 
piano educativo e didattico. Esso costituisce un elemento di riferimento che, se correttamente utilizzato, consente l'osservazione dell'allievo nel corso del suo cammino formativo ed, in un'ottica proattiva, la prevenzione dell'insuccesso. Si compone di quattordici scale valutative, delle quali sette attinenti alla dimensione cognitiva e sette a quella affettivo-motivazionale. È strutturato in 100 item, descriventi un modo di agire, di giudicare le situazioni e se stessi, di vivere emotivamente le varie esperienze in un contesto formativo.

Ai fini orientativi, l'impiego di questo tipo di strumenti assume un valore specifico: consente di stimolare l'auto-riflessione e la presa di coscienza riguardo ad alcuni aspetti spesso poco controllati intenzionalmente, del proprio modo di relazionarsi alle varie situazioni di studio e di vita. Sollecitando, inoltre, lo studente a riflettere sul significato attribuito al proprio percorso di crescita formativa e professionale, focalizza l'attenzione sulla dimensione dell'auto-determinazione, del soddisfacimento delle motivazioni intrinseche e sul possesso da parte del singolo di un progetto esistenziale che lo guidi nelle proprie scelte.

\subsection{Le modalità di somministrazione del QSA}

Nell'ambito delle attività laboratoriali finalizzate a promuovere negli studenti processi di riflessione e auto-valutazione sulle competenze strategiche messe in atto per autodirigersi nello studio e nel lavoro è stato proposto il QSA (Questionario sulle Strategie di Apprendimento, Pellerey, 1996 e 2013). La compilazione del questionario avviene tramite la piattaforma e-learning del corso.

$\mathrm{Al}$ termine della compilazione è restituito allo studente un profilo individuale che riporta in forma grafica, integrata da un commento testuale, l'elaborazione dei punteggi. Quello che segue è un esempio di profilo grafico ottenuto al termine della compilazione del QSA.

Il profilo grafico ottenuto consente di valutare a colpo d'occhio la distribuzione dei punteggi per ciascun fattore e di verificarne il posizionamento nella media oppure sopra o sotto di essa. Basta interpretare la dimensione della barra in colore verde rispetto al valore centrale della scala, segnato da un tratto verticale (Figura 1). Se la barra verde supera il tratto centrale il valore è superiore alla media, se invece si colloca sotto è inferiore. Tuttavia ai fini dell'interpretazione dei risultati, vengono considerati nella media i punteggi che oscillano da 4 a 6 , inferiori alla media i punteggi da 1 a 3 e superiori alla media i punteggi compresi tra 7 e 9 . Poiché alcune scale risultano inverse non per tutti i fattori un punteggio superiore alla media è da considerarsi come un dato positivo. Per facilitare la lettura dei risultati, i fattori sui quali porre attenzione, in quanto possibili indicatori di criticità (C3, C4, C6, A1, A7). 


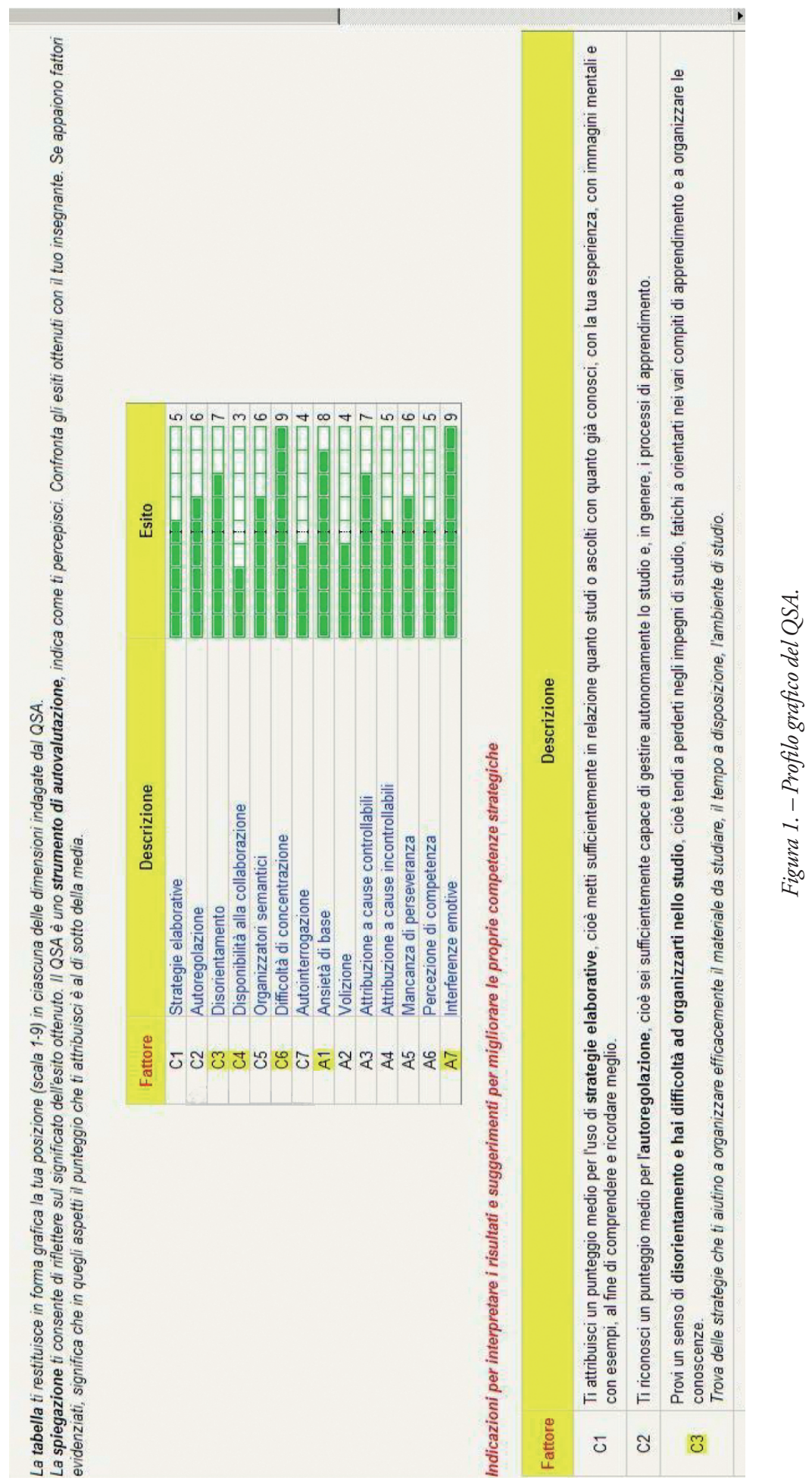


La rappresentazione grafica del profilo, anche per favorire un riscontro immediato da parte dello stesso studente, è completata da un commento di tipo testuale degli esiti. Per ciascuno dei fattori, il commento esplicita il posizionamento rispetto alla scala e fornisce una interpretazione del suo significato con eventuali suggerimenti di carattere compensativo, qualora il punteggio dovesse risultare come critico.

Infine, cliccando sopra la voce sintetica di ciascuno dei fattori è possibile avere una descrizione più dettagliata del fattore stesso e delle sue implicazioni rispetto alle strategie di apprendimento. Il profilo può essere salvato in formato PDF, stampato e inviato direttamente al proprio indirizzo e-mail.

Allo studente è richiesto di interpretare il proprio profilo partendo dal testo che il questionario stesso restituisce. Si tratta di un'analisi di primo livello attraverso la quale lo studente analizza gli esiti per ciascun fattore del questionario. Avvia in tal modo una prima riflessione tanto su aspetti di matura cognitiva quanto affettivo-motivazionale. Sebbene con un'analisi di primo livello lo studente possa già avviare un processo di auto-valutazione, nelle attività laboratoriali si sviluppa un'analisi più approfondita, o di secondo livello, attraverso la quale si richiede di analizzare le reciproche connessioni tra fattori inquadrandoli all'interno di più vaste aree di competenza a partire dallo schema interpretativo della Tabella 1.

Tabella 1. - Aree di competenza e fattori.

\begin{tabular}{ll}
\hline \multicolumn{1}{c}{ AREE DI COMPETENZA } & \multicolumn{1}{c}{ FATTORI } \\
\hline $\begin{array}{l}\text { Gestire processi e strategie elaborative } \\
\text { per comprendere e ricordare }\end{array}$ & C1 Strategie elaborative \\
Crientarsi e organizzarsi nei compiti di studio & C7 Auto-interrogazione \\
& C2 Auto-regolazione \\
& C3 Disorientamento \\
Relazionarsi e collaborare con altri & C6 Difficoltà di concentrazione \\
Controllare e gestire ansietà ed emozioni & A1 Ansietà di base \\
Percepire la propria competenza e locus of control & A7 Interferenze emotive \\
& A6 Percezione di competenza \\
& A4 Attribuzione a causecontrollabili \\
Controllare e proteggere le proprie emozioni & A2 Volizione a cause incontrollabili \\
& A5 Mancanza di perseveranza \\
\hline
\end{tabular}


L'analisi compiuta da ciascuno studente confluisce in una relazione consegnata sulla piattaforma e-learning del corso e discussa in sede d'esame. Dalle relazioni emerge una generale soddisfazione degli studenti: in particolare è apprezzata l'opportunità di riflettere, attraverso il profilo del QSA, su aspetti del proprio modo di agire e percepire se stessi sia in relazione alle strategie di studio sia per le dimensioni affettivo motivazionali che sentono come del tutto trascurate rispetto alle proprie esperienze scolastiche.

Oltre alle analisi individuali dei profili, è stata condotta, a scopo di indagine esplorativa, una elaborazione aggregata delle risposte date ai questionari per evidenziare eventuali criticità ricorrenti rispetto alle dimensioni indagate dal questionario e se emergessero uno o più "profili studente» tipici ed infine se tali profili si correlassero a variabili di profitto ed età.

\subsection{Nota metodologica}

Tutti gli studenti $(\mathrm{N}=327)$ iscritti al primo anno del Corso di Laurea triennale in Scienze dell'Educazione, che hanno frequentato nel primo semestre il corso di Didattica Generale, hanno compilato il QSA.

La distribuzione degli studenti per genere e per classi di età rispecchia le caratteristiche degli iscritti al corso di laurea. Per quanto riguarda il genere, infatti, la presenza femminile è molto numerosa con una percentuale del $95 \%$, mentre la distribuzione per classi di età (Figura 2) mostra una marcata asimmetria positiva. Rispetto all'età la composizione dell'aula è molto eterogenea (il campo di variazione dell'età è compreso tra 19 e 48 anni): un gruppo numeroso di studenti $(31,36 \%)$ è rappresentato da matricole; il 38,76\%, con un'età compresa tra 21 e 22 anni, comprende sia matricole, che hanno ritardato l'iscrizione all'università subito dopo il diploma, sia studenti che iscritti ad anni successivi al primo non hanno ancora sostenuto l'esame; infine una compagine numerosa di studenti (circa il 30\%), con un'età superiore ai 23 anni, è iscritta al corso di laurea per ottenere un secondo titolo oppure si tratta di studenti lavoratori che, in molti casi, già operano nella scuola $o$ nel mondo della formazione, e desiderano completare con la laurea il loro percorso di studi.

Al termine del corso di Didattica Generale, un'alta percentuale di studenti (il 70\%) ha affrontato e superato l'esame nella prima sessione (Figura 3) conseguendo buoni risultati dal momento che circa il 50\% ha ottenuto una valutazione almeno pari a 28/30. 


\section{classi di eta'}

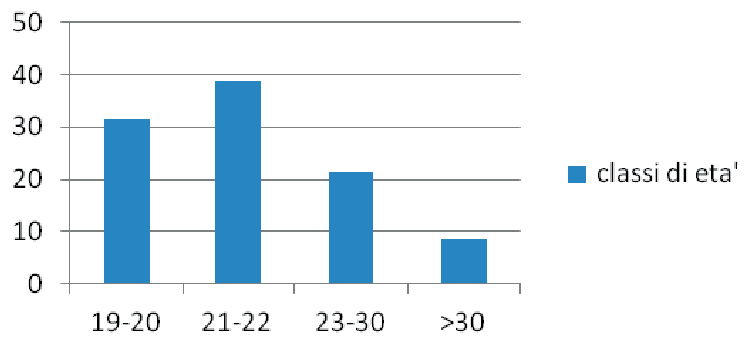

Figura 2. - Grafico a barre della distribuzione delle frequenze percentuali degli studenti per classi di età.

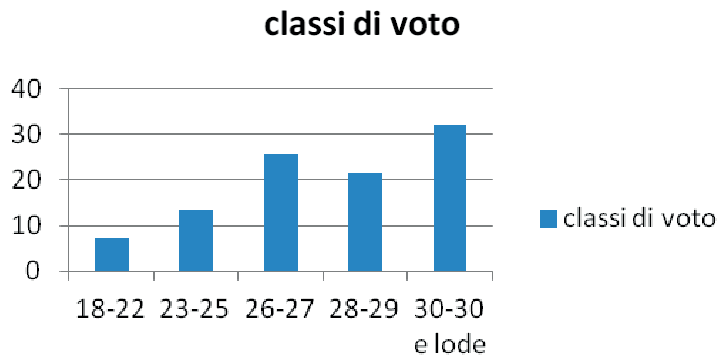

Figura 3. - Grafico a barre della distribuzione delle frequenze percentuali degli studenti per classi di voto.

\subsubsection{Analisi dei risultati del questionario}

In questo articolo si è cercato di utilizzare i risultati del questionario al fine di riuscire a tracciare profili relativi alle competenze strategiche degli studenti che hanno frequentato il corso di Didattica Generale. Seguendo la validazione del questionario effettuata da Pellerey ad ogni fattore viene attribuito un valore compreso tra 1 e 9 secondo una scala stanine. Nell'analisi statistica effettuata, tali valori sono stati raggruppati in 3 classi: valutazione bassa, media e alta, secondo il seguente criterio:

- valori $1,2,3 \rightarrow$ valutazione bassa (valore 1 );

- valori 4, 5, 6 $\rightarrow$ valutazione media (valore 2 );

- valori 7, 8, $9 \rightarrow$ valutazione alta (valore 3 ).

L'analisi dei risultati del questionario, congiuntamente alle variabili «classi di voto» e "classi di età», è stata effettuata utilizzando l'Analisi delle Corri- 
spondenze Multiple (ACM), procedura introdotta negli anni ' 70 , ad opera della scuola francese di analisi dei dati di Benzecri (1982) ${ }^{2}$. L'ACM fa parte delle tecniche fattoriali e ha lo scopo di sintetizzare le relazioni che intercorrono fra numerose variabili sottoposte ad analisi simultanea, allo scopo di riprodurne il patrimonio informativo in un numero più ridotto di variabili sintetiche = fattori. Il presupposto è che le variabili trattate siano associate tra loro e che in virtù di ciò possano esprimere (almeno in parte) uno stesso tipo di informazione. La presenza di correlazione tra le variabili del questionario è stata precedentemente verificata, per motivi di brevità (la matrice delle correlazioni ha dimensione $13 \times 13$ ) i valori delle correlazioni non sono riportate nel presente articolo, possimao tuttavia affermare che solo il $29 \%$ dei coefficienti di correlazione $(\rho)$ è compreso tra $-0,20<\rho<0,20$.

\subsection{I profili studente}

Dall'ACM emergono abbastanza nettamente tre profili di studente con caratteristiche ben delineate, più un quarto profilo che rappresenta invece lo studente medio.

Nel quarto quadrante, in basso a destra (Figura 4) è rappresentato il bravo studente che si caratterizza per una buona capacità di studio (fattori $\mathrm{C} 1$, C5; C7 con valore 3), una buona percezione di competenza (fattore A6 con valore 3) e un locus of control interno (fattore A3 con valore 3), si dimostra inoltre volitivo (fattore A2 con valore 3 ) e con buona competenza autoregolativa (fattore $\mathrm{C} 2$ con valore 3 ).

Nel quadrante 1, in alto a destra, è rappresentato lo studente equilibrato che si caratterizza per la propria solidità emotiva, capace di controllare le proprie emozioni e stati ansiosi (fattori A7 e A1 con punteggio 1), orientato (fattore C3 con punteggio 1), capace di perseverare negli impegni (fattore A5 con punteggio 1), con uno stile attributivo che non lega le ragioni dei propri successi od insuccessi a cause incontrollabili (fattore A4 con punteggio 1) e con buona capacità di mantenere la concentrazione (fattore C6 con punteggio 1).

Nel terzo quadrante, in basso a destra, si colloca invece lo studente disorientato, che si caratterizza per una difficoltà di controllo dei propri stati emotivi (fattori A1 e A7 con punteggio 3) alto disorientamento (fattore C3 con punteggio 3), difficoltà di concentrazione (fattore C6 con punteggio 3), incapace a manifestare perseveranza nei propri impegni (fattore A5 con punteggio 3) e con la convinzione che le ragioni dei propri successi od insuccessi sia determinata da cause che sono fuori dal proprio controllo (fattore A4 con punteggio 3).

\footnotetext{
2 Benzecri, 1982.
} 


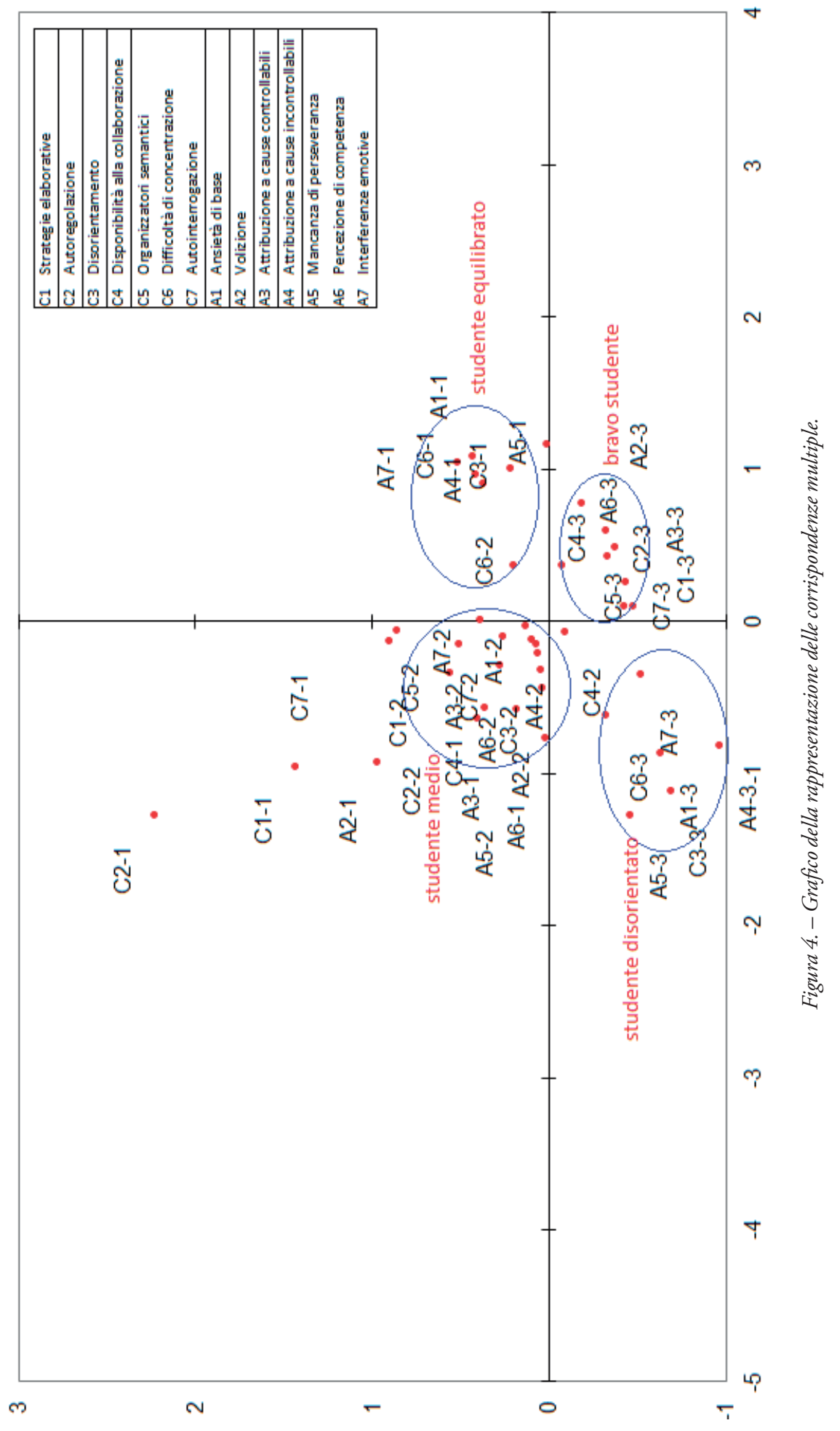


Nel secondo quadrante, quello in alto a sinistra, si colloca il profilo dello studente medio, ossia di uno studente che registra punteggi nella media per tutti i fattori sia di natura cognitiva sia di natura affettivo motivazionale. Quindi nella media per i tre fattori che fanno capo alla capacità di gestire processi e strategie per comprendere e ricordare $(\mathrm{C} 1, \mathrm{C} 5, \mathrm{C} 7)$, per la capacità di organizzarsi nei compiti di studio (fattori C2, C3, C6) con scarsa disponibilità alla collaborazione (fattore $\mathrm{C} 4$ ). Nella madia anche per i fattori affettivo motivazionali: per il controllo e la gestione dell'ansietà e delle proprie emozioni (fattori A1 e A7), la percezione della propria competenza e locus of control (fattori A6, A3 e A4) e sempre nella media volizione e perseveranza (fattori A2, A5).

Sottoponendo, infine, ad ACM anche le variabili età e voto conseguito all'esame, dal grafico ${ }^{3}$ emerge abbastanza chiaramente, come ci si poteva attendere, un'associazione della classe di voto superiore (30 e 30 e lode) con i profili del «bravo studente» e dello «studente equilibrato», così come risulta un'associazione tra il profilo dello studente equilibrato e la classe di età superiore (oltre i 30). Allo "studente medio" risultano invece associate le classi di voto medio e medio alto (dal $25 \mathrm{al} 29)$ e le classi di età più giovani (19-22), mentre allo studente disorientato si associa nettamente la variabile «esame non sostenuto".

\section{APPRENDIMENTO COOPERATIVO ONLINE}

In un articolo recente Johnson, Johnson e Smith (2014) propongono una meta-analisi degli studi sull'apprendimento cooperativo condotti in ambito universitario, in particolare in Nord America, ma anche in Medio Oriente, Europa ed Asia a partire dagli anni' 60 del Novecento fino al primo decennio del XXI secolo.

Confrontando i diversi studi analizzati (305) gli autori rilevano che i risultati hanno indicato che l'apprendimento cooperativo promuove maggiore realizzazione individuale rispetto a quello competitivo (incremento $=0,49) \mathrm{e}$ a quello individualistico (incremento $=0,53$ ).

Gli autori precisano che si tratta di incrementi significativi e sostanziali ed individuano gli elementi della realizzazione individuale nei seguenti descrittori: acquisizione e conservazione della conoscenza, precisione e creatività

3 Il grafico non è inserito nel testo per ragioni di spazio e perché nella stampa, visto il grande numero di variabili interessate, risultano sovrapposti gli indici rendendone difficile la lettura. Chi fosse interessato può farne richiesta agli autori. 
nel problem solving, raggiungimento di un livello superiore di ragionamento. Questi risultati sono stati conseguiti dagli studenti per compiti verbali (come la lettura, la scrittura, e la presentazione orale), operazioni matematiche ed attività fisiche (come nuoto, golf e tennis). Rilevano inoltre che alcuni studi hanno evidenziato un vantaggio, per l'apprendimento cooperativo, nella promozione del pensiero meta-cognitivo, nella disponibilità a svolgere compiti difficili, nell'impegno a dedicare maggiore tempo al compito, nella perseveranza (nonostante eventuali difficoltà) posta nel raggiungimento degli obiettivi, nella motivazione intrinseca, nella capacità di trasferire le nozioni acquisite da una situazione all'altra (Johnson \& Johnson, 1989). Gli autori sottolineano inoltre che questi risultati sono confermati in una meta-analisi svolta tra ricerche che si sono concentrate in particolare nell'analisi dell'applicazione del cooperative learning in corsi di livello universitario di scienza, matematica, ingegneria, e tecnologia (Springer et al., 1999).

Infine è interessante riportare gli studi che mostrano come la qualità della vita universitaria può dipendere in larga misura dalla qualità delle relazioni tra gli studenti e tra studenti e docenti (McKeachie et al., 1986; Astin, 1993; Tinto, 1993; Pascarella, 2001). Le relazioni interpersonali positive possono aumentare la qualità dell'adattamento sociale alla vita universitaria, sviluppare negli studenti il senso di appartenenza intellettuale all'istituzione, ridurre le incongruenze tra gli interessi degli studenti e i curriculi di studio, favorire l'integrazione nella vita accademica e l'impegno a completare gli studi (Tinto, 1993).

La letteratura internazionale mostra che anche le pratiche di tutorato tra pari ${ }^{4}$ in ambito universitario possono influenzare positivamente gli studenti nel mantenere costante la motivazione e l'affettività verso lo studio, contribuire a ridurre il drop-out e favorire una maggiore regolarità nella carriera accademica. Infatti queste strategie didattiche che hanno ottenuto risultati molto positivi nelle scuole primaria e secondaria dove sono esercitate principalmente come sostegno cognitivo agli studenti (Sharpley \& Sharpley, 1981; Cohen et al., 1982; Johnson \& Johnson, 1986; Slavin, 1990; Topping, 2001a; Rohrbeck et al., 2003; Topping, 2005), in ambito universitario producono risultati positivi soprattutto in ambito affettivo-motivazionale e socio-relazionale (Wolfe et al., 1984; Pigott et al., 1986; Griffin \& Griffin, 1997; Fantuzzo \& Ginsburg-Block, 1998; Topping \& Ehly, 1998; Topping et al., 2000; Sluijsmans et al., 2004; Aoun, 2008).

4 Naturalmente l'apprendimento tra pari si basa sulla cooperazione, sull'assistenza e l'incoraggiamento e dunque non si svolge in una situazione di tipo competitivo né tantomeno di tipo individualistico; pertanto il tutorato tra pari (peer tutoring o reciprocal peer tutoring) si sviluppa in una dimensione di cooperative learning. 
Inoltre la ricerca indica che i benefici dell'apprendimento cooperativo possono essere trasferiti negli ambienti virtuali creati nella rete (Calvani, 2005). McConnell (McConnell, 2000; McConnell, 2005; Bradley \& McConnell, 2008; Crosta \& McConnell, 2008) nei suoi molteplici studi sull'argomento, fornisce esempi di sistemi informatici di apprendimento collaborativo costruiti per supportare la comunicazione e la condivisione di documenti in modalità sincrona e asincrona. Naturalmente il successo delle attività in cooperative learning dipendono da una progettazione pedagogicodidattica appropriata che permetta agli studenti di condividere il loro lavoro (Koschmann, 1996) anche in ambito universitario (Strijbos et al., 2004).

All'interno della progettazione di un intervento di apprendimento cooperativo, Smith e Coenders (2002) ritengono necessario prevedere la costruzione di uno strumento che consenta di raccogliere dati sulle attività di lavoro di gruppo online, ed individuano nel cosiddetto community barometer, un questionario da somministrare ai partecipanti, lo strumento per ottenere un feed-back sulla esperienza vissuta (Arnold \& Smith, 2003). Il barometro permette agli studenti di riconoscersi come una comunità di apprendimento online, e ai docenti del corso di ricevere informazioni sul clima instauratosi nel gruppo e quindi offre loro la possibilità di correggere eventuali criticità. Nella letteratura internazionale consultata non risulta la realizzazione di un barometro standardizzato, pertanto la costruzione del barometro utilizzato in questa esperienza si deve alla autrice dello studio; si ritiene interessante la prospettiva di pervenire ad una standardizzazione dello strumento elaborato in un prossimo lavoro di ricerca.

\subsection{Descrizione dello studio: ipotesi, metodologia, strumenti, esiti e criticità}

Lipotesi generale che ha sorretto questa esperienza, e che ha indirizzato lo studio degli esiti prodotti dalle relative attività di monitoraggio e valutazione, sostiene che l'apprendimento collaborativo in ambito universitario contribuisce all'acquisizione, negli studenti, della meta-cognizione necessaria per portare a termine con profitto e successo il proprio percorso di formazione.

Nel merito, si ritiene che l'apprendimento collaborativo online, attivando pratiche di interdipendenza positiva, possa facilitare l'inserimento degli studenti nell'ambiente universitario e, attraverso il potenziamento della motivazione e la riduzione dell'ansia legata all'esame, consentire loro di arginare l'abbandono degli studi e di acquisire maggiore consapevolezza del percorso accademico intrapreso, favorendo così l'orientamento in itinere. 


\subsection{Metodologia e svolgimento dell'esperienza}

Come si è detto nel primo paragrafo, questo lavoro presenta le procedure e gli esiti delle attività di apprendimento cooperativo online, svolte dagli studenti nell'a.a. 2013/14 nell'ambito dell'insegnamento di Didattica Generale (DG); in analogia con lo studio pilota dell'a.a. 2012/13 (La Rocca et al., 2014), questo secondo studio è consistito in una osservazione empirica del processo e del prodotto dell'intervento formativo articolato in due prospettive: il monitoraggio del clima stabilitosi nel gruppo e la rilevazione del risultato ottenuto all'esame.

In particolare si presentano gli esiti ottenuti a seguito della realizzazione delle ipotesi di intervento auspicate nelle considerazioni conclusive del precedente studio, ovvero: estendere l'esperienza ad altri insegnamenti ${ }^{5}$; rendere più agile il questionario, detto barometro ${ }^{6}$, utilizzato per il monitoraggio; somministrare il barometro non solo a termine del corso ma anche nel suo svolgimento per consentire agli studenti un feed-back in itinere e agli osservatori uno sguardo più completo sul clima sviluppatosi nei gruppi, sulle aspettative degli studenti e sulle loro opinioni rispetto all'esperienza vissuta; osservare se in media si è verificato un incremento nei risultati conseguiti dagli studenti nell'esame di Didattica Generale confrontando gli esiti di coloro che hanno partecipato alle attività collaborative con quelli di chi non vi ha preso parte.

Premettendo che l'insegnamento si svolge in modalità blended, in presenza e a distanza, su piattaforma online, e che l'esperienza di apprendimento cooperativo riguarda, appunto, le attività online, si offre, di seguito, una rappresentazione schematica dei percorsi di intervento formativo e di ricerca effettuati nel primo e nel secondo anno, secondo una numerazione che consente di individuare lo sviluppo diacronico e sequenziale delle attività (Tabella 2).

5 L'esperienza è stata di fatto estesa anche all'insegnamento di Strategie Educative e Nuovi Processi Comunicativi (SE) per il Corso di Laurea Magistrale in Educatore Professionale di Comunità, ma in questa sede non se ne dà conto perché si osserva esclusivamente il contesto del corso di Didattica Generale.

6 Come già detto il barometro utilizzato non è uno strumento standardizzato; è stato costruito dall'autrice, naturalmente in riferimento alle indicazioni della relativa letteratura scientifica, e testato sul campo nello studio pilota dello scorso anno accademico; si intende procedere alla standardizzazione dello strumento in un prossimo lavoro di ricerca.

ECPS Journal - 10/2014

http://www.ledonline.it/ECPS-Journal/ 


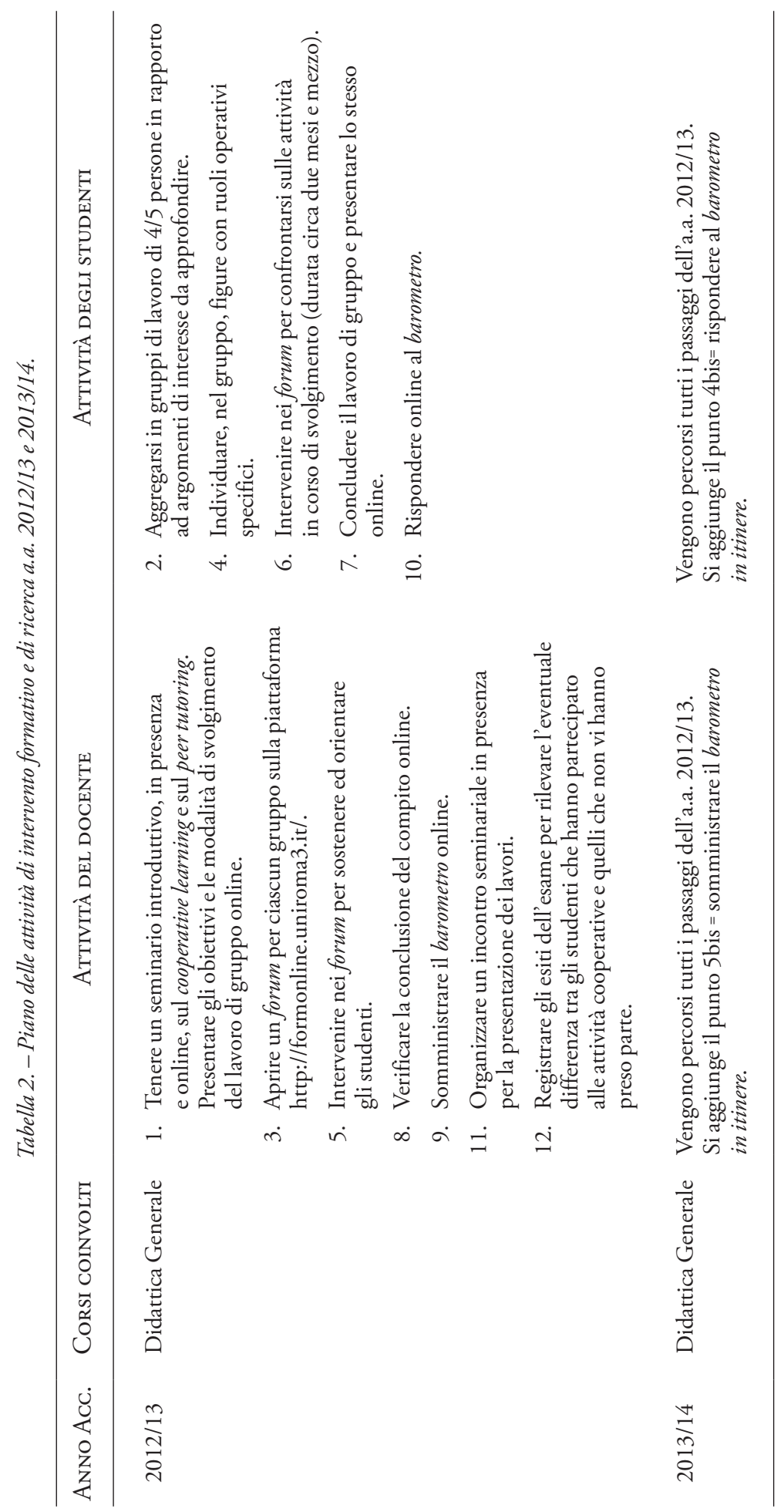


Il docente introduce gli studenti alle attività da svolgere in gruppo presentando sinteticamente le teorie e le strategie didattiche che trattano dell'apprendimento collaborativo, con particolare riferimento al cooperative learning e al peer tutoring. Il seminario è svolto in presenza con il supporto di diapositive e materiali multimediali; gli stessi materiali utilizzati nella lezione sono inseriti in piattaforma in modo che gli studenti, frequentanti e non, possano avere costantemente accesso alle informazioni e ne possano discutere con il docente e tra di loro nel forum aperto allo scopo.

Nell'ambito dell'intervento seminariale, rivolto alla platea degli studenti, il docente indica gli obiettivi, che ciascuno studente, nel lavoro di gruppo, dovrà perseguire, distinguendoli per macro-categorie: gli obiettivi cognitivi e metacognitivi e gli obiettivi affettivi e relazionali. I primi intendono promuovere l'uso di un linguaggio descrittivo adeguato dal punto di vista scientifico-disciplinare, l'individuazione di informazioni significative, l'esecuzione di comparazioni, il confronto di informazioni e dati per analogia e opposizione, l'assunzione di consapevolezza della funzione svolta dal percorso collaborativo nella costruzione di conoscenza, la capacità di sintetizzare materiali in un documento finale collettivo, la riflessione sul proprio contributo alla realizzazione del prodotto finale. Gli obiettivi affettivi e relazionali sono diretti alla acquisizione della capacità di dialogo e di ascolto attivo per comunicare contenuti ed emozioni e per porre in essere un sostegno reciproco nel percorso di apprendimento.

Sempre nel seminario introduttivo, il docente indica i passaggi operativi necessari per la formazione dei gruppi e per l'allestimento delle attività online, ed in presenza. I lavori di gruppo online, sono avviati circa alla metà dello svolgimento del corso, per dar modo agli studenti di acquisire elementi di conoscenza relativi ai contenuti disciplinari e di familiarizzare tra loro online, ed in presenza.

Considerando il contesto universitario i gruppi sono costituiti, in maniera autonoma, dagli stessi studenti in base ad interessi condivisi e nel rispetto delle indicazioni fornite nel seminario introduttivo, ovvero: non superare le cinque unità per gruppo; individuare un argomento da approfondire e da sottoporre all'approvazione del docente; decidere quale denominazione attribuirsi; individuare uno studente con funzioni di coordinatore e uno che assuma il compito di redattore del lavoro finale.

Gli studenti comunicano le proprie scelte al docente attraverso la piattaforma del corso di studi - http://formonline.uniroma3.it/ - negli spazi riservati all' "Insegnamento di Didattica Generale» dove il docente, per ciascun gruppo, apre un forum in cui i ragazzi possano dialogare e scambiarsi materiali e idee online; sono previste figure tutoriali rivestite da cultori della materia pronti ad intervenire al bisogno, ma attenti a non indebolire la dimensione del peer tutoring, anzi promuovendola ed incoraggiandola, come pares inter pares. 
Una volta terminate le operazioni di costituzione dei gruppi, della scelta degli argomenti da approfondire, dell'attribuzione dei forum e dei tutor, viene comunicata agli studenti la data prevista per dare avvio allo studio di gruppo da svolgersi e terminare entro i tempi stabiliti. Infine si individua la giornata seminariale per la presentazione, alla platea degli studenti, del documento prodotto da ciascun gruppo consistente o in un iper-testo, o in un PowerPoint, o in un testo wiki o in una mappa concettuale interattiva.

\subsection{Monitoraggio delle attività}

Il monitoraggio è stato effettuato attraverso la somministrazione di un breve questionario utilizzato come barometro ${ }^{7}$; il questionario, come un barometro, ha lo scopo di cogliere la "pressione» e il "clima» che si sono generate nel gruppo di lavoro, raccogliendo le opinioni degli studenti riguardo alla esperienza di attività collaborativa vissuta. La letteratura scientifica indica nel barometro uno strumento per la rilevazione dell'auto-percezione del clima nel gruppo; esso è generalmente utilizzato per restituire un feed-back al docente e agli stessi studenti partecipanti in merito alle modalità relazionali e alle azioni produttive avvenute nel gruppo. Poiché in letteratura vengono altresì riportati casi in cui tale strumento sembra essere stato recepito dai partecipanti al gruppo come elemento di disturbo o di intrusione ${ }^{8}$, nella prima esperienza, avvenuta lo scorso anno, si decise di somministrare il barometro solo al termine del processo formativo. Come è stato anticipato al par. 3.2, in questa seconda esperienza si è deciso di proporre il barometro agli studenti in due momenti, dopo circa due settimane dall'avvio delle attività di gruppo e al termine delle stesse. Questa doppia somministrazione ha permesso di effettuare un confronto tra le aspettative che gli studenti riponevano nelle attività collaborative e le opinioni maturate dopo l'effettivo svolgimento delle stesse.

$\grave{E}$ inoltre necessario sottolineare che nella prima esperienza il barometro è stato costruito con una preminenza di domande aperte per consentire maggiore libertà di espressione e dunque per raccogliere indicazioni utili per l'allestimento di uno strumento più agile e strutturato da utilizzare nelle future indagini omologhe 9 . Per ottenere comunque dati comparabili, si è proceduto

7 Jones, 2002; Smith \& Coenders, 2002; Svensson et al. (1999), cit. in S. Mazzucconi, Uno strumento per l'autovalutazione dei gruppi collaborativi: il "barometro», in Calvani, 2005.

8 Ibidem.

9 Il barometro somministrato nello scorso a.a. 2012/13 era formato da 10 quesiti: i primi tre (A, B, C) sono strutturati con quattro risposte predeterminate articolate secondo una Scala Likert a quattro livelli (Molto, Abbastanza, Poco, Per niente); gli altri (D, E, F, G, H, I, L) sono quesiti aperti con risposte libere; i questionari analizzati sono stati 48. La codifica e 
ad una riconfigurazione delle domande aperte, trasformando in alternative di risposta chiusa le risposte libere precedentemente fornite dagli studenti. $\mathrm{Si}$ è mantenuta comunque l'opzione della alternativa di risposta aperta per tutte le domande, per consentire agli studenti eventuali risposte libere. Il $b a$ rometro in itinere contiene le stesse domande di quello in uscita, con l'unica variante delle forme temporali che esprimono l'aspettativa nel primo e la affermazione di dati di fatto osservati e vissuti nel secondo.

Il barometro somministrato consta di 10 quesiti ${ }^{10}$ : i primi tre (A, B, C) sono strutturati e ciascuno prevede risposte predeterminate articolate secondo una scala Likert a quattro livelli (1: Molto, 2: Abbastanza, 3: Poco, 4: Per niente); gli altri (D, E, F, G, H, I, L) sono quesiti strutturati con multiple alternative di scelta, derivate dalle risposte libere fornite dagli studenti lo scorso anno.

Nel merito, il quesito A sollecita una riflessione sul contesto relazionale; il B sui contenuti di apprendimento; il C sulla gestione del lavoro; i quesiti D (che si articola in D1, D2, D3) ed E sulle abilità ritenute necessarie per affrontare l'esperienza; le domande F, G, H sul ruolo del singolo nel gruppo e sul rapporto tra singolo e gruppo; i quesiti I ed L richiedono di indicare eventuali criticità e di offrire suggerimenti per il miglioramento dell'esperienza. La totalità delle alternative di risposta predefinite, e distribuite variamente per ciascuna domanda, è di 61.

Come per lo scorso anno la rilevazione degli esiti dell'esame di Didattica Generale è stata focalizzata sull'osservazione del voto d'esame ottenuto dagli studenti partecipanti alle attività collaborative in rapporto alla valutazione ottenuta dagli studenti che non vi hanno partecipato. Per riassumere, dunque, questo secondo studio è consistito in una osservazione empirica del processo e del prodotto dell'intervento formativo articolato in due prospettive: il monitoraggio, attraverso il barometro, del clima stabilitosi nei gruppi di lavoro formatisi nell'ambito degli insegnamenti di DG e SE e la rilevazione del risultato ottenuto all'esame di Didattica Generale.

\subsection{Descrizione e commento dei risultati}

Il numero dei questionari restituiti ed analizzati è di 63 per quello in itinere e di 67 per l'uscita ${ }^{11}$; la codifica e decodifica delle risposte è stata realizzata

decodifica delle risposte è stata realizzata in SPSS, con procedure di codificazione a posteriori per macro-categorie per le risposte alle domande aperte e con procedimenti convenzionali di tipo quantitativo per le risposte alle domande chiuse.

10 Per motivi di spazio non è possibile inserire lo strumento; può essere richiesto a concetta. larocca@uniroma3.it.

11 Si veda supra, p. 248. 
in SPSS, con procedure di codificazione a posteriori per macro-categorie per le risposte alle domande aperte e con procedimenti convenzionali di tipo quantitativo per le risposte alle domande chiuse.

Sono state effettuate le seguenti elaborazioni di statistica descrittiva: analisi univariata delle singole risposte per i questionari in itinere ed in uscita per permettere il confronto diacronico tra aspettative ed esiti; analisi bivariata tra le risposte fornite a coppie di domande solo per i questionari in uscita per osservare, sincronicamente, l'esito effettivo dell'esperienza in rapporto a specifici temi; analisi multivariata solo per i questionari in uscita per focalizzare su un sistema di assi cartesiani la posizione assunta dalle risposte ad alcuni item selezionati secondo una specifica prospettiva.

I risultati ${ }^{12}$ ottenuti dalla somministrazione del barometro sono stati generalmente molto positivi e, tranne che per alcuni casi che verranno evidenziati, non hanno presentato grandi scollamenti tra le somministrazioni in ingresso e in uscita, ovvero tra le aspettative verso l'apprendimento cooperativo e la realizzazione delle attività in gruppo.

In particolare, in rapporto alle domande $\mathrm{A}$ e $\mathrm{B}^{13}$ gli studenti hanno ritenuto che le attività collaborative abbiano consentito la costruzione di relazioni di interdipendenza positiva, migliorato il rapporto con il docente del corso e determinato una disposizione favorevole nei confronti dell'esame. Inoltre hanno espresso l'opinione che sarebbe interessante praticare attività di lavoro di gruppo anche in altri insegnamenti poiché la ricerca dei materiali è risultata utile ed interessante e le attività di approfondimento hanno potenziato l'apprendimento visto che il lavoro di gruppo li ha "costretti» a riflettere sui propri processi di comprensione.

Ė interessante osservare che, nell'ambito di DG, alla alternativa «Sarebbe interessante praticare lavori di gruppo anche in altri insegnamenti» della domanda B, il barometro in uscita ha registrato un incremento di 3,4 punti percentuali: evidentemente gli studenti di DG, essendo matricole, hanno trovato nel lavoro di gruppo un sostegno che sperano di poter ritrovare in altri corsi.

Per quanto riguarda la domanda $\mathrm{C}$, che interroga gli studenti sulla gestione del lavoro di gruppo, è interessante notare che l'alternativa che, nel $b a$ rometro in itinere, proponeva la "Competizione» come elemento che avrebbe potuto svilupparsi nel corso della attività, è diminuita di 21,3 punti percentuali: gli studenti hanno sperimentato che, nella pratica, lavorare in gruppo può non generare competizione.

12 Per motivi di spazio non si riportano i dati numerici analitici e i relativi grafici; il materiale può essere richiesto a concetta.larocca@uniroma3.it.

${ }_{13}$ La somma delle percentuali delle risposte Molto e Abbastanza oscillano in un range tra l' $85 \%$ e il 100\%, sia per il questionario in itinere che per quello in uscita. 
Alla domanda D gli studenti, in grandissima maggioranza, hanno identificato le abilità cognitive necessarie a lavorare in gruppo nella consapevolezza delle proprie competenze e nella condivisione delle proprie conoscenze; le abilità relazionali nell'ascolto dell'altro e nel rispetto delle idee altrui per costruire un clima di collaborazione che consenta di organizzare il lavoro e di gestire positivamente i conflitti anche attraverso l'impegno emotivo, la fiducia, la determinazione e il senso di responsabilità. In questo caso nell'ambito di DG lo scarto più significativo tra la risposte in itinere e quelle in uscita è stato rilevato in incremento per la abilità "Consapevolezza delle proprie competenze» $(3,3 \%)$ e "Fiducia» $(4,8 \%)$ ed in decremento per l'abilità "Gestione dei conflitti» (13\%) e "Pazienza» $(4,5 \%)$; i ragazzi pensavano che lavorare in gruppo avrebbe richiesto uno sforzo perseverante nel gestire i conflitti, ed hanno invece appurato che servono più la fiducia e pazienza. In risposta alla domanda E, la maggior parte degli studenti ritiene che le abilità per lavorare in gruppo possano essere acquisite grazie ad esperienze simili a quella vissuta ed hanno rilevato come all'interno di ciascun gruppo ognuno abbia svolto il proprio compito in maniera differenziata assumendosi le responsabilità negoziate con i compagni di lavoro.

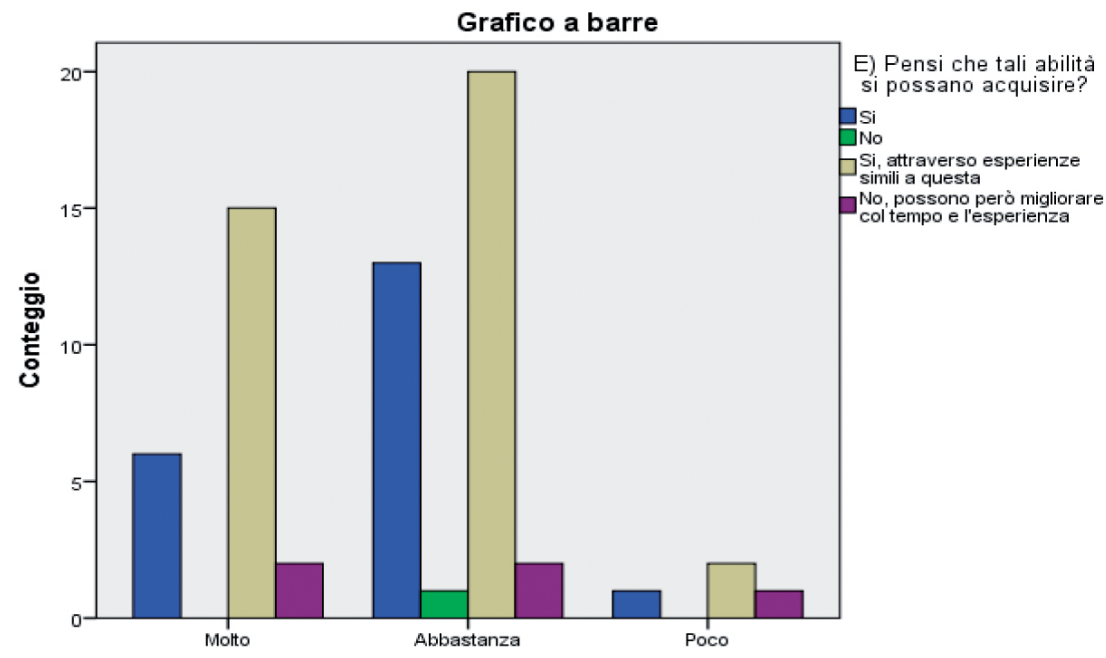

D1.2 Tra le abilità che pensi sia necessario possedere per lavorare in gruppo, indica quanto conta la "condivisione delle proprie conoscenze"

Figura 5. - Grafico a barre multiple

relativo alle domande D ed E - "Barometro» in uscita - Didattica Generale. 
Si è effettuata una analisi bivariata tra ciascuna delle alternative di risposta previste nella domanda $\mathrm{D}$ e le risposte alla domanda $\mathrm{E}$ : l'esito è stato pressoché lo stesso per ogni incrocio considerato, ovvero la percentuale maggiore di risposte positive ha mostrato che, per ciascuna delle abilità indicate, gli studenti hanno ritenuto che si sarebbe potuta acquisire e sviluppare attraverso esperienze simili a quella vissuta, ovvero attraverso esperienze di cooperative learning. Nella Figura 5, a sinistra si riporta, come esempio, il grafico relativo all'incrocio tra la domanda D1.2 e la domanda E, per il barometro in uscita.

In risposta alla domanda $\mathrm{H}$, che chiede di esprimere la propria opinione sullo studiare in gruppo o da soli, gli studenti, per la maggior parte, hanno indicato di preferire lo studio collettivo per ottimizzare i tempi di lavoro, per scambiare idee ed opinioni, per acquisire consapevolezza dei contenuti che si padroneggiano e di quelli da approfondire; alcuni hanno sottolineato che lo studio individuale non è in alternativa allo studio in gruppo, e può essere preferibile nel caso in cui si debba affrontare una prova che richieda particolare elaborazione personale. In particolare, nello scarto temporale tra i due barometri, nell'insegnamento di DG si è registrato, in uscita, un incremento in rapporto alla preferenza di lavorare in gruppo per "Avere consapevolezza dei contenuti padroneggiati» (13,7\%), "Confrontarsi e scambiare idee ed opinioni» (5,9\%) ed un decremento notevole rispetto a «In genere preferisco lavorare da solo ma questa esperienza mi aiuterà / $\mathrm{mi}$ ha aiutato a saper collaborare» (22\%). Sembra dunque che l'esperienza fatta sia stata maggiormente soddisfacente rispetto alla condivisione di contenuti e alla riflessione su di essi piuttosto che dal punto di vista della dimensione relazionale, infatti nelle alternative di risposta che riguardano questo aspetto non si è verificato un incremento rilevante. Sembra interessante proporre uno sguardo incrociato tra le risposte alle domande I ed L, ponendo dunque in relazione la richiesta della domanda I di esprimere eventuali criticità emerse nell'esperienza vissuta e della L di offrire possibili relativi suggerimenti.

Dall'analisi del grafico (Figura 6) è evidente che la grande maggioranza $(67,2 \%)$ degli studenti non ha rilevato criticità nell'esperienza di apprendimento cooperativo vissuta; si rileva che tali criticità sono emerse in particolare in rapporto alla scelta dei componenti del gruppo poiché i ragazzi ritengono che non tutti abbiano svolto la stessa mole di lavoro. $\grave{E}$ interessante osservare che in due opzioni sull'asse delle ascisse compare l'indicazione di potenziare il ruolo del tutor e/o del docente sia per aiutare il gruppo a gestire la distanza fisica, sia per offrire un valido sostegno nel superare eventuali difficoltà incontrate. 


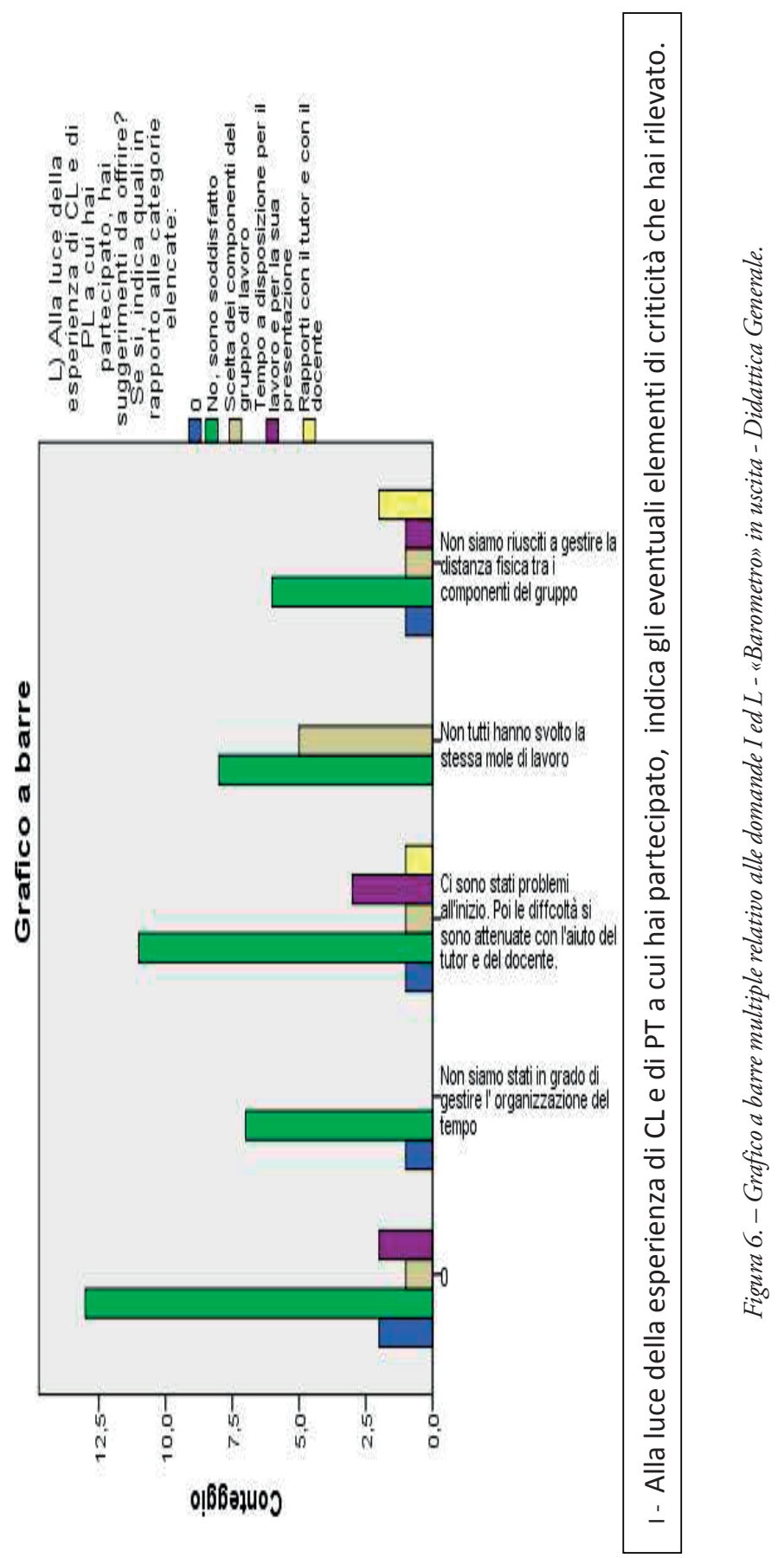


In linea con l'esperienza effettuata lo scorso anno, è sembrato interessante porsi l'interrogativo di come gli studenti abbiano interpretato, messo in pratica e vissuto l'impegno di organizzare e gestire autonomamente, seppure con il sostegno del tutor, il gruppo di lavoro. Per tale motivo si è deciso di effettuare una analisi delle corrispondenze multiple tra gli item che richiedevano considerazioni inerenti a "L'organizzazione del lavoro nel gruppo». I risultati dell'analisi sono riportati nella Figura 7.

Le lettere e i numeri rappresentati sul piano cartesiano corrispondono rispettivamente agli item e alle alternative di risposta; per favorire la comprensione della interpretazione che verrà proposta, nella casella di fianco al grafico sono riportate per esteso le domande e le risposte che corrispondono ai punti contenuti nei cerchi (es: F.3 = Item F, alternativa di risposta 3, oppure C4.1 = Item C, alternativa di risposta 4, Scala Likert1).

Osservando il grafico, una prima evidenza riguarda la densità dell'aggregazione in prossimità del punto di origine degli assi, intorno al quale si sono collocate le valutazioni positive espresse dalla maggioranza degli studenti, i quali hanno ritenuto che l'esperienza di cooperative learning online abbia sviluppato collaborazione, crescita di abilità relazionali, abbia prodotto un lavoro finale soddisfacente; hanno inoltre affermato di essersi sentiti valorizzati in un contesto dove ciascuno si è assunto le proprie responsabilità impegnandosi nel lavoro.

Si notano altre due piccole zone di aggregazione, la prima nel quadrante I in alto a destra, la seconda nel quadrante IV in prossimità dell'asse delle ordinate; la posizione delle due aggregazioni mostra che il primo gruppo di punti, lontano dalla zona centrale, rappresenta risposte decisamente negative, mentre il secondo gruppo, che si colloca a ridosso dell'asse delle ordinate e più vicino alla zona occupata da quelle positive, rappresenta le risposte che si attestano su posizioni di moderata criticità. Le risposte decisamente negative esprimono la posizione di quei pochi studenti che ritengono che questa esperienza abbia favorito la competizione più che la collaborazione e che il lavoro nel gruppo sia stato gestito molto male. Per approfondire il dato, si è effettuata una analisi qualitativa isolando nei dati su foglio SPSS le specifiche risposte negative e si è verificato che essi appartenevano allo stesso gruppo di lavoro. Quindi si è analizzato il forum aperto sulla piattaforma in cui gli studenti appartenenti a questo gruppo hanno postato i propri interventi. Da questa osservazione è risultato che i tre ragazzi hanno iniziato con entusiasmo e sollecitudine le attività richieste, individuando il tema da approfondire, distribuendosi i compiti e hanno proseguito con la raccolta di numerosi materiali multimediali; nella fase conclusiva, quella più propriamente operativa nella quale bisognava arrivare alla stesura finale del lavoro, sono emersi alcuni problemi che hanno impedito loro di portare a termine le attività e di presentare il proprio prodotto nel seminario finale. 


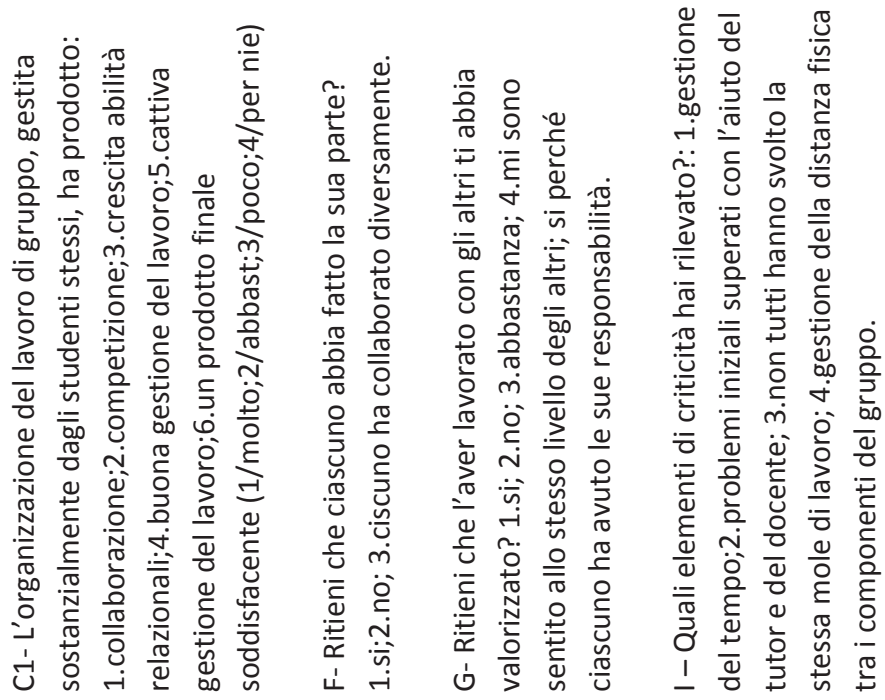

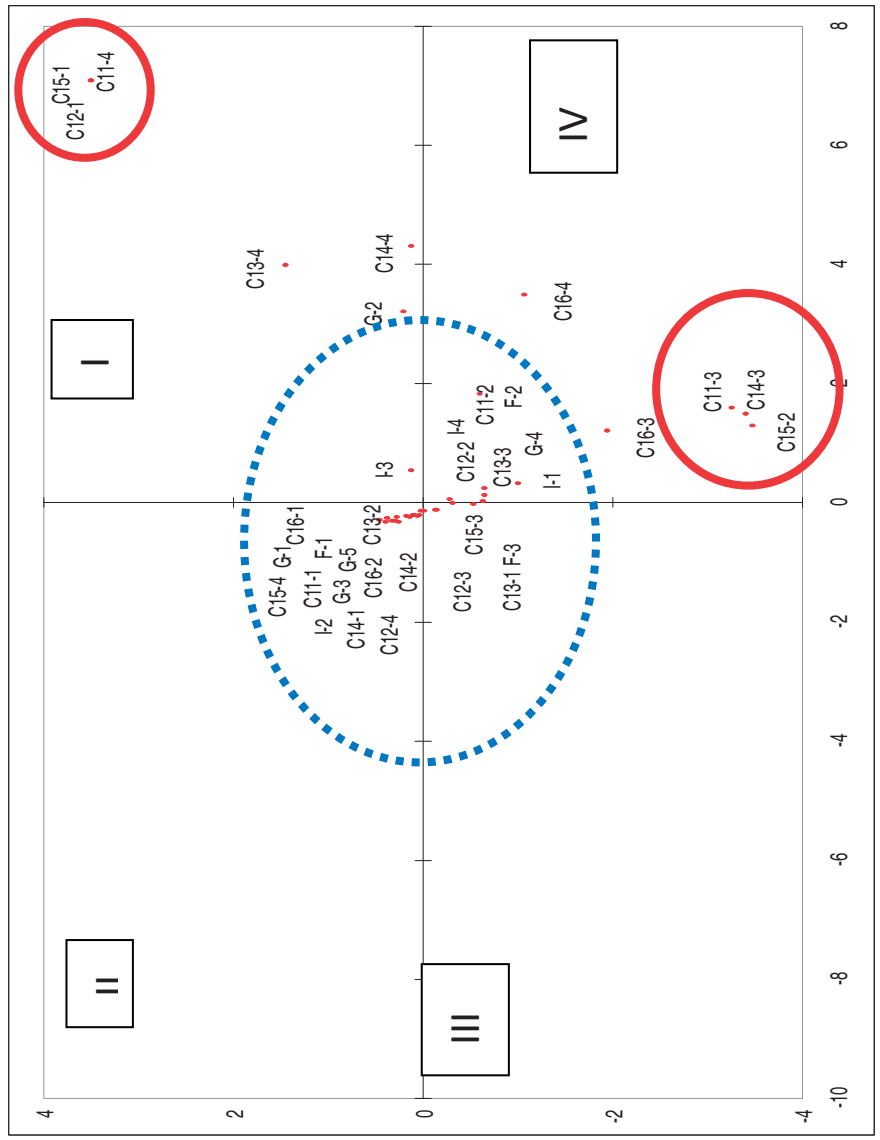


In particolare una di loro non ha svolto la sua parte di lavoro impedendo anche agli altri di raggiungere l'obiettivo previsto. Probabilmente questo spiega il motivo per il quale abbiano espresso giudizi così fortemente negativi sull'esperienza vissuta.

La lettura della ACM (Analisi delle Corrispondenze Multiple) sembra confermare l'informazione assunta anche dalle analisi univariata e bivariata, dalle quali è emersa una certa problematicità nel vissuto nell'insegnamento di DG soprattutto in merito alla difficoltà di gestire i rapporti tra i componenti del gruppo sia dal punto di vista relazionale che da quello relativo alla assunzione di responsabilità individuale nello svolgimento del lavoro.

La Figura 8 mostra l'esito della rilevazione del risultato ottenuto all'esame di Didattica Generale, per il quale, come si è detto, si è osservato il voto all'esame ottenuto dagli studenti partecipanti alle attività collaborative in rapporto alla valutazione degli studenti che non hanno partecipato alle attività di gruppo.

Come per lo scorso anno, è necessario sottolineare che l'ottica assunta per lo studio statistico dei dati è stata puramente descrittiva ed esplorativa dal momento che è stata presa in considerazione la popolazione degli studenti che hanno partecipato ai gruppi collaborativi e la popolazione degli studenti che non vi hanno partecipato, naturalmente in riferimento all'a.a. 2013/14. Dal confronto dei box-plot (Figura 8) è possibile rilevare non soltanto che il voto medio, conseguito all'esame di Didattica Generale dagli studenti che hanno partecipato alle attività di gruppo risulti mediamente più alto di quelli che non vi hanno partecipato, ma anche che nel secondo gruppo è presente una variabilità minore tra i voti conseguiti da ciascuno studente, in rapporto

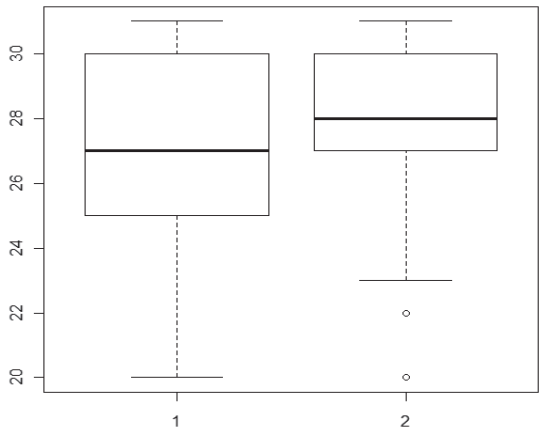

1: studenti che non hanno partecipato ai gruppi $(\mathrm{n} 1=150$ studenti, voto medio $=27$; deviazione standard $=2,71$ )

2 : studenti che hanno partecipato ai gruppi (n2 =78 studenti, voto medio $=28$, deviazione standard $=2,45)$

Figura 8. - Risultato dell'esame di Didattica Generale 2013/14. 
al primo gruppo che risulta molto meno coeso da questo punto di vista poiché il voto minimo conseguito dagli studenti del $2^{\circ}$ gruppo è " 23 " mentre quello conseguito dal secondo gruppo è «20», essendo 30 il punteggio massimo.

\section{CONCLUSIONI, CRITICITÀ E PROSPETTIVE FUTURE}

La sperimentazione delle attività di auto-valutazione delle proprie competenze strategiche attraverso il QSA e di cooperative learning online effettuate nell'a.a. 2013/14 è risultata sostanzialmente molto positiva, come lo era stata l'esperienza pilota dell'a.a 2012/13.

Molti studenti, matricole al primo esame, attraverso il QSA hanno avuto l'opportunità di riflettere sulle proprie strategie di apprendimento e sull'importanza di pianificare, attraverso comportamenti autoregolati, i propri impegni universitari. Si è osservato a tal proposito che un'alta percentuale di studenti ha affrontato l'esame con successo nella prima sessione $(70 \%$ circa). Inoltre, con l'opportunità di svolgere attività in cooperazione, gli studenti si sono sentiti maggiormente coinvolti nel percorso di apprendimento e nella costruzione stessa dell'insegnamento attraverso i contribuiti elaborati nei lavori di approfondimento in gruppo.

Gli studenti hanno verificato che il lavoro di gruppo contribuisce a sviluppare abilità cognitive e meta-cognitive, oltre che abilità relazionali, e ritengono che esperienze simili a quella vissuta possano essere auspicabili nel proprio percorso accademico. Inoltre le operazioni di analisi, sintesi, valutazione e correzione online, dei percorsi seguiti e dei materiali proposti, oltre all'esposizione pubblica in presenza del lavoro finale, hanno contribuito a rafforzare in essi l'autostima, il senso di appartenenza ad una comunità che apprende e la disposizione positiva verso la disciplina e l'esame di profitto.

Pur all'interno di una esperienza sicuramente positiva sia nella gestione che negli esiti, si è rilevato comunque un elemento di criticità nel non essere riusciti ad elaborare i dati del barometro in itinere in tempo utile per fornire un feed-back agli studenti sull'andamento delle attività di cooperative learning online; di fatto i risultati ottenuti dalla somministrazione dei barometro in itinere e in uscita sono stati utili esclusivamente per l'osservazione scientifica dell'esperienza.

Un ulteriore elemento di criticità investe un aspetto della procedura metodologica: poiché il barometro è anonimo, non è stato possibile effettuare un'analisi delle corrispondenze tra gli esiti rilevati con questo strumento e 
il QSA, in particolare nell'aspetto riguardante lo sviluppo delle competenze relazionali e collaborative. Poiché, come si è detto, la compilazione del QSA è obbligatoria per sostenere l'esame di Didattica Generale, mentre le attività di cooperative learning online sono facoltative, per il prossimo a.a. 2014/15 dovranno prevedersi particolari accorgimenti formali che possano permettere l'analisi delle corrispondenze congiunte dei due strumenti.

Inoltre per il futuro sarebbe auspicabile l'inserimento in altri insegnamenti della compilazione del QSA e delle attività di cooperative learning online per contribuire a formare gli studenti alla auto-regolazione nell'apprendimento, alla cooperazione, all'ascolto attivo, alla condivisione di conoscenze e competenze, oltre che per offrire loro un effettivo spazio di aggregazione e di confronto su una piattaforma e-learning, considerando che spesso le lezioni in presenza non facilitano conoscenza e condivisione a causa della numerosità dei frequentanti e della impossibilità di molti alla frequenza.

\section{RIFERIMENTI BIBLIOGRAFICI}

Aoun, C. (2008). Peer-assessment and learning outcomes: product deficiency or process defectiveness? Educational Assessment Annual Conference, Cambridge, September. http://www.iaea2008.cambridgeassessment.org.uk/ca/digitalAssets/180447_ Aoun.pdf.

Arnold, P., \& Smith, J. (2003). Adding connectivity and losing context with ICT: Contrasting learning situations from a community of practice perspective. In M. Huysman, E. Wenger, \& V. Wulf (Eds.), Communities and technologies (pp. 465-484). Proceedings of the first International Conference on communities and technologies. Dordrecht: Kluwer Academic.

Astin, A. (1993). What matters in university: Four critical years revisited. San Francisco: Jossey-Bass.

Bandura, A. (2000). Autoefficacia: teoria e applicazioni. Trad. it. a cura di G. Lo Iacono (ed. orig. 1997). Trento: Erikson.

Bay, M., Grzadziel, D., \& Pellerey, M. (2010). Promuovere la crescita nelle competenze strategiche che hanno le loro radici nelle dimensioni morali e spirituali della persona. Rapporto di ricerca. Roma: CNOS FAP.

Benzecri, J.-P. (1982). L’analyse des données. Leçons sur l'analyse factorielle et la reconnaissance des formes et travaux. Paris: Dunod.

Boscolo, P. (1981). Intelligenza e differenze individuali. In AA.VV., Intelligenza e diversità. Torino: Loescher. 
Bradley, S. A., \& McConnell, D. (2008). Virtual groups in learning environments: Collaboration, cooperation or (self) centred individualism? In V. Hodgson et al. (Eds.), Proceedings of the 6th International Conference on networked learning (pp. 24-31). http://www.networkedlearningconference.org.uk/abstracts/ PDFs/Bradley_24-31.pdf.

Cainarca, G. C., \& Sgobbi, F. (2005). Educational mismatch e skill mismatch: un'indagine empirica sui lavoratori italiani, XX Convegno Nazionale di economia del lavoro, Roma, Università «La Sapienza», 22-23 Settembre.

Calvani, A. (2005). Rete, comunità e conoscenza. Costruire e gestire dinamiche collaborative. Trento: Erickson.

Crosta, L., \& McConnell, D. (2008). Online learning groups development: A grounded international comparison. InV. Hodgson et al. (Eds.), Proceedings of the 6th International Conference on networked learning (pp. 61-68). http:// www.networkedlearningconference.org.uk/.

Cohen, P. A., Kulik, J. A., \& Kulik, C. C. (1982). Educational outcomes of tutoring: A meta-analysis of findings. American Educational Research Journal, 19, 237-248.

D’Alessio, M., Laghi, F., \& Pallini, S. (2006). Mi oriento. Il ruolo dei processi motivazionali e volitivi. Padova: Piccin.

Elias, P., \& McKnight, A. (2001). Skill measurement in official statistics: Recent developments in the UK and the rest of Europe. Oxford Economic Papers, 53, 508540.

Fantuzzo, J., \& Ginsburg-Block, M. (1998). Recirpocal peer tutoring: Devleoping and testing effective peer collaborations for elementary school students. In K. Topping \& S. Ehly, Peer-assisted learning (pp. 121-145). Mahwah, NJ: Lawrence Erlbaum Associates Publishers.

Griffin, B. W., \& Griffin, M. M. (1997). The effects of reciprocal peer tutoring on graduate student achievement, test anxiety, and academic self-efficacy. The Journal of Experimental Education, 65(3), 197-209.

Johnson, D. W., Johnson, R. T., \& Smith, K. (2014). Cooperative learning: Improving university instruction by basing practice on validated theory. In N. Davidson, C. Major, \& L. Michaelsen (Eds.), Small-group learning in higher education: Cooperative, collaborative, problem-based and team-based learning. Journal on Excellence in College Teaching, 25(4). http://personal. cege.umn.edu/ smith/docs/Johnson-Johnson-Smith-Cooperative_LearningJECT-Small_Group_Learning-draft.pdf.

Johnson, D. W., \& Johnson, R. (1989). Cooperation and competition: Theory and research. Edina, $\mathrm{MN}$ : Interaction Book Company.

Johnson, D. W., \& Johnson, R. T. (1986). Learning together and alone (2nd ed.). Englewood Cliffs, NJ: Prentice Hall. 
Kolb, D. A. (1985). LSI Learning Style Inventory: Self-Scoring inventory and interpretation booklet. Boston: McBer and Co.

Koschmann, T. (Ed.). (1996). CSCL: Theory and practice of an emerging paradigm. Mahwah, NJ: Lawrence Erlbaum Associates Publishers. http://www. networkedlearningconference.org.uk/abstracts/PDFs/Crosta_61-68.pdf.

La Rocca, C. (2009). Mediazione tutoriale e apprendimento in rete. Il tutor organizzativo e disciplinare nella didattica e.learning. Roma: Monolite.

La Rocca, C. (2012). Il Peer Tutoring nell'orientamento universitario. Punti di forza e criticità. MeTis, 2(1).

La Rocca, C., Margottini, M., \& Capobianco, R. (2014). Collaborative learning in higher education. Open Journal of Social Sciences.

McConnell, D. (2005). Examining the dynamics of networked e.learning groups and communities. Studies in Higher Education, 30(1), 23-40.

Lazarus, R. S., \& Folkman, S. (1984). Stress, appraisal and coping. New York: Springer.

McConnell, D (2000). Implementing computer supported cooperative learning (2nd ed.). Kogan Page.

McKeachie, W., Pintrich, P., Yi-Guang, L., \& Smith, D. (1986). Teaching and learning in the university classroom: A review $f$ the research literature. Ann Arbor, MI: The Regents of the University of Michigan.

Pascarella, E. (2001). Cognitive growth in university. Change, 33(6), 21-27.

Pellerey, M. (2006). Dirigere il proprio apprendimento. Brescia: La Scuola.

Pellerey, M., \& Orio, F. (1996). Questionario sulle strategie d'apprendimento (QSA). Con 25 schede e floppy disk. Roma: Las.

Pellerey, M., Epifani, F., Grządziel, D., Margottini, M., \& Ottone, E. (in corso di stampa). Imparare a dirigere se stessi. Progettazione e realizzazione di una guida $e$ di uno strumento informatico per favorire l'autovalutazione e lo sviluppo delle proprie competenze strategiche nello studio e nel lavoro.

Piaget, J. (1985). The equilibration of cognitive structures. Chicago: University of Chicago Press.

Pigott, H. E., Fantuzzo, J. W., \& Clement, P. W. (1986). The effects of reciprocal peer tutoring and group contingencies on the academic performance of elementary school children. Journal of Applied Behavior Analysis, 19, 93-98.

Rohrbeck, C. A., Ginsburg-Block, M. D., Fantuzzo, J. W., \& Miller, T. R. (2003). Peer-assisted learning interventions with elementary school students: A metaanalytic review. Journal of Educational Psychology, 95, 240-257.

Salmon, G. (2004a). E-tivities. The key to active online learning. London: Routledge Falmer.

Salmon, G. (2004b). E-moderating. The key to teaching and learning online. London: Routledge Falmer. 
Savickas, M. (2005). The theory and practice of career construction. In S. D. Brown $\&$ R. W. Lent (Eds.). Career development and counseling. Putting theory and research to work (pp. 42-70). New York: John Wiley - Hoboken.

Serbati, A., Zaggia, C. (2012). Allineare le metodologie di insegnamento, apprendimento e valutazioneai learning outcomes: una proposta per i corsi di studio universitari. Giornale Italiano della Ricerca Educativa, 5, Dicembre, 11-26.

Sharpley, A. M., \& Sharpley, C. F. (1981). Peer tutoring: A review of the literature. Collected Original Resources in Education, 5(3), 7-C11.

Slavin, R. E. (1990). Co-operative learning: Theory, research and practice. Englewood Cliffs, NJ: Prentice Hall.

Sluijsmans, D. M. A., Brand-Gruwel, S., Van Merrienboer, J. J. G, \& Martens, R. L. (2004). Training teachers in peer assessment skills: Effects on performance and perceptions. Innovation in Education and Teaching International, 41(1), 59-78.

Smith, J., \& Coenders, M. (2002). E-feedback to reflect legitimate peripheral participation; towards a redefinition of feedback in online learning environments. In M. Driscoll \& T. Reeves (Eds.), Proceedings of World Conference on e-learning in corporate, government, healthcare, and higher education (pp. 878-890). Chesapeake, VA: Association for the Advancement of Computing in Education.

Springer, L., Stanne, M. E., \& Donovan, S. S. (1999). Effects of small-group learning on undergraduates in science, mathematics, engineering, and technology: A meta-analysis. Review of Educational Research, 69(1), 212-251.

Stahl, G., Koschmann, T., \& Suthers, D. (2006). Computer-supported collaborative learning: An historical perspective. In R. K. Sawyer (Ed.), Cambridge handbook of the learning sciences (pp. 409-426). Cambridge: Cambridge University Press. http://gerrystahl.net/cscl/CSCL_English.pdf.

Strijbos, J.-W., Kirschner, P., \& Martens, R. (Eds.). (2004). What we know about CSCL ... And implementing it in higher education. Dordrecht, Netherlands: Kluwer Academic Publishers. http://link.springer.com/chapter/10.1007\%2F14020-7921-4_1\#page-2.

Tinto, V. (1993). Leaving university: Rethinking the causes and cures of student attrition (2nd ed.). Chicago: University of Chicago Press.

Topping, K. J. (2001). Peer assisted learning: A practical guide for teachers. Cambridge, MA: Brookline Books.

Topping, K. J. (2005). Trends in peer learning. Educational Psychology, 25(6), 631645.

Topping, K. J., \& Ehly, S. (1998). Peer-assisted learning. Mahwah, NJ: Lawrence Erlbaum Associates Publishers.

Topping, K. J., Smith, E. F., Swanson, I., \& Elliot, A. (2000). Formative peer assessment of academic writing between postgraduate students. Assessment \& Evaluation in Higher Education, 25(2), 149-166. 
Vygotskij, L. (1987). Il processo cognitivo. Torino: Bollati Boringhieri.

Wolfe, J. A., Fantuzzo, J. W., \& Wolter, C. F. (1984). Student-administered grouporiented contingencies: A method of combining group-oriented contingencies and self-directed behavior to increase academic productivity. Child and Family Behavior Therapy, 6, 45-60.

Yerkes, R. M., \& Dodson, J. D. (1908). The relation of strength of stimulus to rapidity of habit-formation. Journal of Comparative Neurology and Psychology, $18,459-482$.

Yin Robert, K. (2005). Lo studio di caso nella ricerca scientifica. Progetto e metodi. Roma: Armando.

Zimmerman, B. J. (2000). Attaining self-regulation: A social cognitive perspective. In M. Boekaerts, P. R. Pintrich, \& M. Zeidner (Eds.), Handbook of self-regulation (pp. 13-39). San Diego, CA: Academic Press.

Zimmerman, B. J. (2001). Achieving academic excellence: A self-regulatory perspective. In M. Ferrari (Ed.), The pursuit of excellence through education (pp. 85-110). Mahwah, NJ: Lawrence Erlbaum Associates Publishers.

Zimmerman, B. J. (2002). Achieving self-regulation: The trial and triumph of adolescence. In F. Pajares \& T. Urdan, Academic motivation of adolescents (pp. 1-27). Greenwich, CT: IAP.

\section{Riassunto}

Le ricerche mostrano che nella didattica universitaria risulta sempre più diffuso l'utilizzo di ambienti digitali in integrazione alla didattica in presenza. In questo lavoro si considera il contributo che può essere offerto dagli ambienti online per sviluppare negli studenti una disposizione positiva verso il percorso accademico, cosi da promuovere lo sviluppo di competenze trasversali, favorire l'auto-regolazione e dunque migliorare la regolarità degli studi $e$ arginare l'abbandono. In particolare verranno prese in considerazione le attività svolte online nell'a.a. 2013/14 dagli studenti del Corso di Studio di Didattica Generale istituito presso il Dipartimento di Scienze della Formazione dell'Università "Roma Tre». Tali attività riguardano la realizzazione di laboratori per lo sviluppo di competenze autoregolative attraverso la somministrazione del QSA (Questionario per le Strategie di Apprendimento) e per lo sviluppo di competenze trasversali attraverso la partecipazione a lavori di gruppo strutturati. Si è osservato che gli studenti, attraverso il QSA, hanno avviato riflessioni sulle proprie strategie di apprendimento e sull'importanza di pianificare gli impegni universitari: un dato positivo in tale senso è l'alta percentuale di studenti che hanno affrontato l'esame con successo nella prima sessione (70\% circa). Inoltre, dai dati rilevati da questionari (detti "barometro»), si evince che gli studenti hanno verificato che il lavoro di gruppo contribuisce a sviluppare abilità cognitive e meta-cognitive; in particolare risulta 
non soltanto che il voto medio, conseguito all'esame di Didattica Generale dagli studenti in apprendimento cooperativo risulti mediamente più alto rispetto agli altri, ma anche che il «sigma» calcolato nel primo gruppo è inferiore a quello del secondo.

Parole chiave: Ambienti digitali, Apprendimento cooperativo, Competenze trasversali, Orientamento delle matricole, QSA (Questionario per le Strategie di Apprendimento). 